\title{
Spin wave theory of one-dimensional generalized Kitaev model
}

\author{
Wang Yang, ${ }^{1}$ Alberto Nocera, ${ }^{1}$ and Ian Affleck ${ }^{1}$ \\ ${ }^{1}$ Department of Physics and Astronomy and Stewart Blusson Quantum Matter Institute, \\ University of British Columbia, Vancouver, B.C., Canada, V6T $1 Z 1$
}

\begin{abstract}
In this work, we perform a combination of classical and spin wave analysis on the one-dimensional spin- $S$ Kitaev-Heisenberg-Gamma model in the region of an antiferromagnetic Kitaev coupling. Four phases are found, including a Néel ordered phase, a phase with $O_{h} \rightarrow D_{3}$ symmetry breaking, and " $D_{3}$-breaking I, II" phases which both break $D_{3}$ symmetries albeit in different ways, where $O_{h}$ is the full octahedral group and $D_{3}$ is the dihedral group of order six. The lowest-lying spin wave mass is calculated perturbatively in the vicinity of the hidden $\mathrm{SU}(2)$ symmetric ferromagnetic point.
\end{abstract}

\section{INTRODUCTION}

Frustration in low dimensional strongly correlated magnetic systems leads to a plethora of fascinating behaviors 1 [ An unusual way of introducing magnetic frustrations is by strong spin-orbit couplings, which induce bond- and direction-dependent magnetic interactions 9 -11. A famous example of frustrated magnetic system of this type is the two-dimensional (2D) Kitaev model on the honeycomb lattice ${ }^{12}$. The model was proposed to host exotic fractionalized excitations including Majorana fermions and anyons ${ }^{12}$, and has triggered tremendous research interests in recent years 13 난. The 2D Kitaev model can be realized in Mott insulating $\mathrm{A}_{2} \mathrm{IrO}_{4}(\mathrm{~A}=\mathrm{Li}, \mathrm{Na})$ compounds and $\alpha-\mathrm{RuCl}_{3}$ systems. In real materials, additional symmetry allowed couplings also appear. The generalized Kitaev model has been proposed to describe the real systems ${ }^{911132}$, which includes Heisenberg and Gamma interactions in addition to the Kitaev coupling.

Since quantum fluctuations are enhanced by reducing the spatial dimension, exotic behaviors are expected to emerge also in one-dimensional (1D) strongly spinorbit coupled quantum magnetic systems. A series of recent works have performed both analytical and numerical studies on the phase diagram of 1D spin- $1 / 2$ generalized Kitaev model $\$ 43$. The two-leg ladder case has also been analyzed ${ }^{47 / 48}$, which already shows a similar phase diagram with the $2 \mathrm{D}$ case ${ }^{48}$. In particular, in Ref. 46, the phase diagram of the 1D spin-1/2 Kiteav-HeisenbergGamma chain has been studied in detail, which reveals a rich phase diagram with eleven distinct phases.

In this work, we perform a combination of classical and spin wave analysis on the $1 \mathrm{D}$ spin- $S$ Kitaev-HeisenbergGamma model with an antiferromagnetic (AFM) Kitaev coupling. The phase diagram is shown in Fig. 1. The Néel and " $D_{3}$-breaking I, II" phases for the spin-1/2 case found in Ref. 46 are also confirmed for higher spins. On the other hand, the classical analysis predicts an $O_{h} \rightarrow D_{3}$ symmetry breaking for $J=0$, which is in contrast with the $O_{h} \rightarrow D_{4}$ symmetry breaking for the spin- $1 / 2$ case as discussed in Ref. 44. Our DMRG numerics provide evidence for the $O_{h} \rightarrow D_{3}$ symmetry breaking for $S=1$ and $3 / 2$, based on which we conjecture that the spin- $1 / 2$ case is the only exception where strong quantum fluctuations invalidate the classical analysis.

We have also constructed the spin wave theory which captures the small fluctuations around the classical configurations. The lowest-lying spin wave mass $m_{1}$ is calculated perturbatively in the "Néel", " $O_{h} \rightarrow D_{3}$ " and " $D_{3}$ breaking I" phases close to the hidden $\mathrm{SU}(2)$ symmetric ferromagnetic (FM) FM2 point in Fig. 1. Interestingly, although $m_{1} \propto(K-\Gamma)^{2}$ in the " $O_{h} \rightarrow D_{3}$ " phase (where $J=0)$ and $m_{1} \propto J^{2}$ in the " $D_{3}$-breaking I" phase for $K=\Gamma$, the former requires a second order symplectic perturbation calculation, whereas to obtain the latter, one has to go to third order perturbation, where $K, \Gamma$ and $J$ represent the Kitaev, Gamma and Heisenberg couplings, respectively. In the " $D_{3}$-breaking II" phase, we encounter intrinsic difficulties in the perturbative calculation of the spin wave mass, and $m_{1}$ is studied numerically. The origin of such difficulty is worth further explorations. Finally, we emphasize that the phase diagram in Fig. 1 possibly can only be trusted in a neighborhood of the FM2 point. When approaching the origin of Fig. 1 (i.e., the AFM Kitaev point), enhanced quantum fluctuations arising from frustrations may destroy the classical order.

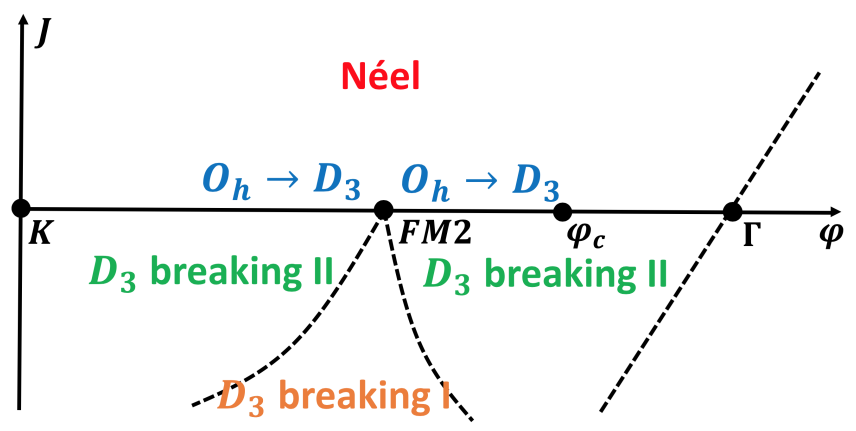

FIG. 1: Classical phase diagram in the vicinity of the FM2 point. The horizontal coordinate $\varphi$ is defined through $K=$ $\cos (\varphi), \Gamma=\sin (\varphi)$. The $\varphi$-coordinates of $K, \mathrm{FM} 2$ and $\Gamma$ points when $J=0$ are $0, \pi / 4$ and $\pi / 2$, respectively. The classical phase transition at $\Gamma$ is shifted to $\varphi_{c}$ by quantum fluctuations. 
(a)

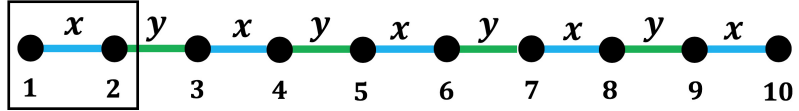

(b)

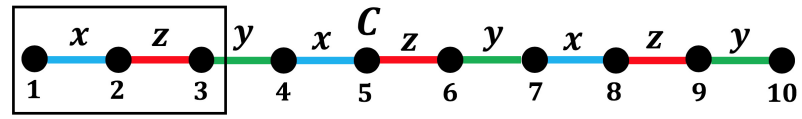

FIG. 2: Bond structures (a) before and (b) after the sixsublattice rotation. The rectangular boxes denote unit cells.

\section{MODEL HAMILTONIAN}

\section{A. The Hamiltonian}

The spin- $S$ Kitaev-Heisenberg-Gamma $(K H \Gamma)$ chain 11 is defined as

$$
H=\sum_{<i j>\in \gamma \text { bond }}\left[K S_{i}^{\gamma} S_{j}^{\gamma}+J \vec{S}_{i} \cdot \vec{S}_{j}+\Gamma\left(S_{i}^{\alpha} S_{j}^{\beta}+S_{i}^{\beta} S_{j}^{\alpha}\right)\right]
$$

in which $\langle i j>$ is used to denote that $i, j$ are nearest neighboring lattice sites; $\gamma=x, y$ is the spin direction associated with the $\gamma$ bond shown in Fig. 2 (a); $\alpha \neq \beta$ are the two remaining spin directions other than $\gamma ; K, J$, and $\Gamma$ are the Kitaev, Heisenberg, and Gamma couplings, respectively; and the spin operators satisfy $\sum_{\alpha=x, y, z}\left(S_{i}^{\alpha}\right)^{2}=S(S+1)$. Since $R(\hat{z}, \pi)$ changes the sign of $\Gamma$ but leaves $K$ and $J$ invariant, there is the equivalence 46

$$
(K, J,-\Gamma) \simeq(K, J, \Gamma),
$$

where the notation $R(\hat{n}, \alpha)$ is used to represent a global spin rotation around the $\hat{n}$-direction by an angle $\alpha$. Parametrizing $K$ and $\Gamma$ as

$$
K=\cos (\varphi), \Gamma=\sin (\varphi),
$$

it is enough to consider $\varphi \in[0, \pi]$ due to the equivalence in Eq. (2). Occasionally, we also use the following parametrization

$$
K=\sin (\theta) \cos (\varphi), \quad \Gamma=\sin (\theta) \sin (\varphi), \quad J=\cos (\theta) .
$$

In this work, we will be interested in the region with an antiferromagnetic Kitaev coupling, i.e., $\varphi \in[0, \pi / 2]$. In particular, we mainly study the region in the vicinity of the FM2 point in Fig. 1 where the coordinates of FM2 are $\varphi=\pi / 4, J=0$ (i.e., $\theta=\pi / 2$ ). Here we note that the notation "FM2" is chosen in accordance with Ref. 46.

A particularly useful six-sublattice rotation $U_{6}$ is defined as $44 \mid 49$

$$
\begin{aligned}
& \text { Sublattice 1: }(x, y, z) \rightarrow\left(x^{\prime}, y^{\prime}, z^{\prime}\right), \\
& \text { Sublattice 2: }(x, y, z) \rightarrow\left(-x^{\prime},-z^{\prime},-y^{\prime}\right), \\
& \text { Sublattice 3: }(x, y, z) \rightarrow\left(y^{\prime}, z^{\prime}, x^{\prime}\right), \\
& \text { Sublattice } 4:(x, y, z) \rightarrow\left(-y^{\prime},-x^{\prime},-z^{\prime}\right), \\
& \text { Sublattice 5: }(x, y, z) \rightarrow\left(z^{\prime}, x^{\prime}, y^{\prime}\right), \\
& \text { Sublattice } 6:(x, y, z) \rightarrow\left(-z^{\prime},-y^{\prime},-x^{\prime}\right),
\end{aligned}
$$

in which "Sublattice $i "(1 \leq i \leq 6)$ represents the collection of the sites $\{i+6 n\}_{n \in \mathbb{Z}}$, and $S^{\alpha}\left(S^{\prime \alpha}\right)$ is abbreviated as $\alpha\left(\alpha^{\prime}\right)$ for short, where $\alpha=x, y, z$. The transformed Hamiltonian $H^{\prime}=U_{6} H U_{6}^{-1}$ acquires the form

$$
\begin{gathered}
H^{\prime}=\sum_{<i j>} \in \boldsymbol{\epsilon \text { bond }}\left[-K S_{i}^{\gamma} S_{j}^{\gamma}-\Gamma\left(S_{i}^{\alpha} S_{j}^{\alpha}+S_{i}^{\beta} S_{j}^{\beta}\right)\right. \\
\left.-J\left(S_{i}^{\gamma} S_{j}^{\gamma}+S_{i}^{\alpha} S_{j}^{\beta}+S_{i}^{\beta} S_{j}^{\alpha}\right)\right],
\end{gathered}
$$

in which the bond $\gamma=x, z, y$ is periodic under translation by three sites as shown in Fig. 2 (b), and the prime has been dropped in $\vec{S}_{i}^{\prime}$ for simplicity. The explicit form of $H^{\prime}$ is included in Appendix A. It is clear from Eq. (6) that the FM2 point is $\mathrm{SU}(2)$ invariant in the six-sublattice rotated frame with an FM coupling.

In the remaining parts of the paper, we will stick to the six-sublattice rotated frame from here on unless otherwise stated.

\section{B. Review of the symmetries}

In this section, we give a quick review of the symmetries of the model within the six-sublattice rotated frame.

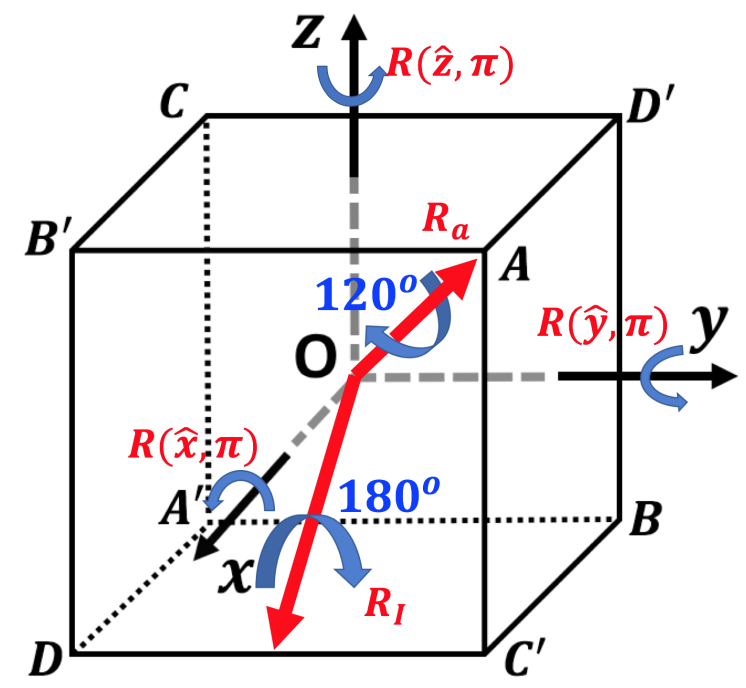

FIG. 3: Actions of the elements in $G /\left\langle T_{3 a}\right\rangle$ in spin space as symmetry operations of a cube.

We first consider the $J=0$ case, i.e., the KitaevGamma chain. The symmetry group has been discussed in detail in Ref. 44. The symmetry transformations include:

1. $T:\left(S_{i}^{x}, S_{i}^{y}, S_{i}^{z}\right) \rightarrow\left(-S_{i}^{x},-S_{i}^{y},-S_{i}^{z}\right)$

2. $R_{a} T_{a}:\left(S_{i}^{x}, S_{i}^{y}, S_{i}^{z}\right) \rightarrow\left(S_{i+1}^{z}, S_{i+1}^{x}, S_{i+1}^{y}\right)$

3. $R_{I} I \quad:\left(S_{i}^{x}, S_{i}^{y}, S_{i}^{z}\right) \rightarrow\left(-S_{10-i}^{z},-S_{10-i}^{y},-S_{10-i}^{x}\right)$

4. $R(\hat{x}, \pi):\left(S_{i}^{x}, S_{i}^{y}, S_{i}^{z}\right) \rightarrow\left(S_{i}^{x},-S_{i}^{y},-S_{i}^{z}\right)$

5. $R(\hat{y}, \pi):\left(S_{i}^{x}, S_{i}^{y}, S_{i}^{z}\right) \rightarrow\left(-S_{i}^{x}, S_{i}^{y},-S_{i}^{z}\right)$

6. $R(\hat{z}, \pi):\left(S_{i}^{x}, S_{i}^{y}, S_{i}^{z}\right) \rightarrow\left(-S_{i}^{x},-S_{i}^{y}, S_{i}^{z}\right)$, 
in which $T$ is time reversal; $T_{a}$ is translation by one lattice site; $I$ is the spatial inversion around the point $C$ in Fig. 2 (b); and $R_{a}=R\left(\hat{n}_{a},-2 \pi / 3\right), R_{I}=R\left(\hat{n}_{I}, \pi\right)$ where

$$
\hat{n}_{a}=\frac{1}{\sqrt{3}}(1,1,1)^{T}, \hat{n}_{I}=\frac{1}{\sqrt{2}}(1,0,-1)^{T} .
$$

We note that the inversion center $C$ can be chosen modulo three. The symmetry group $G$ is generated by the above transformations as

$$
G=<T, R_{a} T_{a}, R_{I} I, R(\hat{x}, \pi), R(\hat{y}, \pi), R(\hat{z}, \pi)>.
$$

Since $T_{3 a}=\left(R_{a} T_{a}\right)^{3}$ is an abelian normal subgroup of $G$, we can consider the quotient group $G /\left\langle T_{3 a}\right\rangle$. It has been worked out in Ref. 44 that the quotient group is isomorphic to $O_{h}$, where $O_{h}$ is the full octahedral group which is the symmetry group of a cube. There is an intuitive understanding of this isomorphism. Neglecting the spatial components in the operations, the actions in spin space are all symmetries of a spin cube as shown in Fig. 3. It is proved in Ref. 44 that the isomorphism still holds even if the spatial components are also included. Hence we conclude that

$$
G \cong O_{h} \ltimes 3 \mathbb{Z},
$$

where $3 \mathbb{Z}=\left\langle T_{3 a}>\right.$ and $\ltimes$ is the semi-direct product.

Next we consider the $J \neq 0$ case, i.e., a general KitaevHeisenberg-Gamma chain. In this case, the system is no longer invariant under the operations $R(\hat{\alpha}, \pi)$ ( $\alpha=$ $x, y, z)$. Thus the symmetry group $G_{1}$ is

$$
G_{1}=<T, R_{a} T_{a}, R_{I} I>.
$$

It has been shown in Ref. 45 that the group structure of $G_{1}$ is

$$
G_{1} \cong D_{3 d} \ltimes 3 \mathbb{Z},
$$

in which $<T, R_{a} T_{a}, R_{I} I>/<T_{3 a}>\cong D_{3 d}$ is used.

\section{Summary of the classical phase diagram}

Here we give a brief summary on the classical phase diagram as shown in Fig. 1 .

The system has a long-range Néel order for $J>0$ where Néel refers to the original frame ${ }^{46}$. In the sixsublattice rotated frame, the "center of mass" directions of the three spins in a unit cell are along $\pm \hat{n}_{a}$-directions as shown by the two solid light blue circles in Fig. 4, where $\hat{n}_{a}$ is defined in Eq. (8). For $|\Delta|,|J| \ll 1$, the lowestlying spin wave mass is calculated to be $\left(\frac{4}{81} \Gamma \Delta^{2}+\frac{2}{3} J\right) S$, where $\Delta=(K-\Gamma) / \Gamma$.

When $J=0$, the ground states are eight-fold degenerate with an $O_{h} \rightarrow D_{3}$ symmetry breaking. Our DMRG numerics provide evidence for the $O_{h} \rightarrow D_{3}$ symmetry breaking for $S=1$ and $3 / 2$, though the spin- $1 / 2$ case is different which has an $O_{h} \rightarrow D_{4}$ symmetry breaking

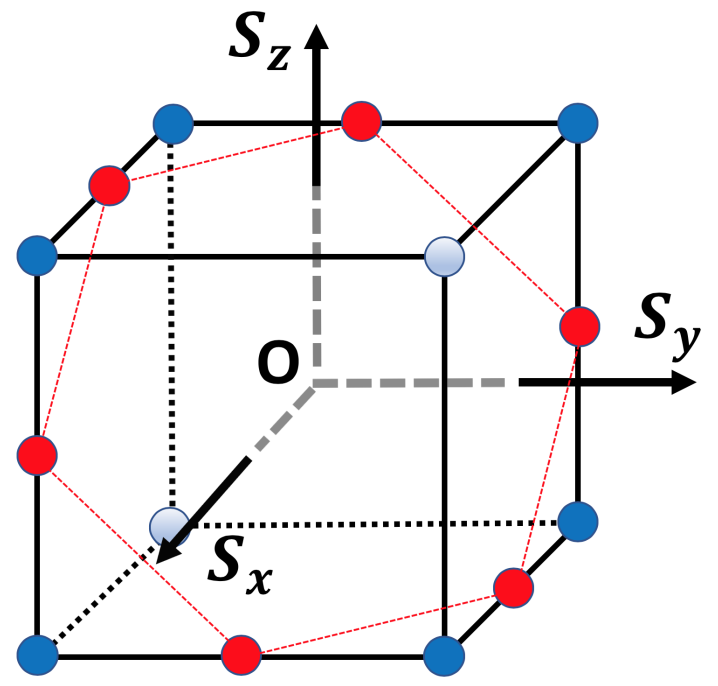

FIG. 4: "Center of mass" directions of the three spins within a unit cell in the six-sublattice rotated frame as represented by: the eight solid blue circles for the eight degenerate ground states in the " $\mathrm{O}_{h} \rightarrow D_{3}$ " phase; the two solid light blue circles (along the $\pm \hat{n}_{a}$-directions) for the two degenerate ground states in the Néel phase; the six solid red circles for the six degenerate ground states in the " $D_{3}$-breaking I" phase; the six solid dark blue circles (removing the two light blue ones among the eight) for the six degenerate ground states in the " $D_{3}$-breaking II" phase. In the " $D_{3}$-breaking II" phase, the plots are for $J \rightarrow 0$ according to the classical analysis. In this paper, the convention of the coordinates is taken such that the eight vertices of the cube are located at $( \pm 1, \pm 1, \pm 1)$.

as discussed in Ref. 44. The "center of mass" spin directions of a unit cell in the eight degenerate $O_{h} \rightarrow D_{3}$ ground states are shown by the eight solid blue circles in Fig. 4. The classical phase transition point for $J=0$ is located at the $\Gamma$-point (i.e., $\varphi=\pi / 2$ ), which is shifted to a different point $\varphi_{c}$ due to quantum fluctuations. For $|\Delta| \ll 1$, the lowest-lying spin wave mass is calculated to be $\frac{4}{81} S \Gamma \Delta^{2}$.

When $J<0$, there are two phases, namely " $D_{3}$ breaking I, II", both having six-fold degenerate ground states. The symmetry breaking patterns of the two phases are $D_{3 d} \rightarrow \mathbb{Z}_{2}^{(\mathrm{I})}$ and $D_{3 d} \rightarrow \mathbb{Z}_{2}^{(\mathrm{II})}$, respectively, where $\mathbb{Z}_{2}^{(\mathrm{I})}$ and $\mathbb{Z}_{2}^{(\mathrm{II})}$ are two different symmetry groups albeit both isomorphic to $\mathbb{Z}_{2}$. In the " $D_{3}$-breaking I" phase, the "center of mass" spin directions of a unit cell in the six degenerate ground states within the six-sublattice rotated frame are plotted as the six solid red circles in Fig. 4. We have calculated the lowest-lying spin wave mass $m_{1}$ for $\Delta=0,|J| \ll 1$ and the result is $S J^{2} / \Gamma$. Although $m_{1}$ is proportional to $J^{2}$, it requires a third order symplectic perturbation calculation as discussed in Sec. VB2 In the " $D_{3}$-breaking II" phase, the "center of mass" spin directions in the six degenerate ground states in the limit $J \rightarrow 0$ are plotted as the six solid dark blue circles in Fig. 4. For larger $|J|$, the "center of mass" directions are distorted away from the vertices of 
the cube. Due to intrinsic difficulties in doing perturbation in the " $D_{3}$-breaking II" phase, we are not able to obtain a perturbative expression for the spin wave mass. On the other hand, the lowest-lying spin wave mass has been studied numerically as shown in Fig. 9. We note that our DMRG numerics provide evidence for the spin ordering patterns in both " $D_{3}$-breaking I, II" phases for $S=1,3 / 2$.

Finally we make a comment on the numerical methods that we employ in this work. The DMRG method was used on chains with length of $L=18$ sites and periodic boundary conditions within the six-sublattice rotated frame. The calculation of the first ten eigenstates was performed using standard DMRG multitargeting approaches 51 . Even though it is known that DMRG convergence is hard for periodic boundary conditions, we have checked that for the system size considered our results are converged using up to $\mathrm{m}=1000$ states with a truncation error below $10^{-6}$ as in previous investigations 4446 .

\section{THE " $O_{h} \rightarrow D_{3}$ " PHASE FOR $J=0$}

In this section, we perform a combination of classical and spin wave analysis for $J=0$ in the vicinity of the FM2 point in Fig. 1. In Sec. IIIA, the trial classical solution is demonstrated to be a minimum of the classical free energy by showing that the eigenvalues of the Hessian matrix are all positive. In Sec. IIIB the symmetry breaking pattern of the classical solution is shown to be $O_{h} \rightarrow D_{3}$, exhibiting an eight-fold degeneracy. Then in Sec. IIIC we derive the spin wave theory by quantizing the Gaussian fluctuations around the classical minima in the long wavelength limit. The smallest spin wave mass is shown to be $\frac{4}{81} S \Gamma \Delta^{2}$ up to the leading nonvanishing order in $\Delta$. Finally in Sec. IIID we provide numerical evidence for the " $O_{h} \rightarrow D_{3}$ " symmetry breaking for $S=1$ and $S=3 / 2$. We work in the six-sublattice rotated frame throughout this section unless otherwise stated.

\section{A. The classical solutions}

The classical analysis is the saddle point approximation in the spin path integral formalism which is valid in the large- $S$ limit. In what follows, we neglect quantum fluctuations of the spins and approximate them as classical three-vectors, i.e.,

$$
\vec{S}_{i}=S \hat{n}_{i},
$$

in which $S$ is the spin magnitude, and $\hat{n}_{i}=\left(x_{i}, y_{i}, z_{i}\right)^{T}$ is a unit vector. The classical free energy of a general $K H \Gamma$ chain is

$$
f=\sum_{n}\left(f_{1+3 n}+f_{2+3 n}+f_{3+3 n}\right),
$$

in which

$$
\begin{gathered}
f_{1+3 n}=-(K+J) S^{2} x_{1+3 n} x_{2+3 n}-\Gamma S^{2}\left[y_{1+3 n} y_{2+3 n}\right. \\
\left.+z_{1+3 n} z_{2+3 n}\right]-J S^{2}\left[y_{1+3 n} z_{2+3 n}+z_{1+3 n} y_{2+3 n}\right], \\
f_{2+3 n}=-(K+J) S^{2} z_{2+3 n} z_{3+3 n}-\Gamma S^{2}\left[x_{2+3 n} x_{3+3 n}\right. \\
\left.+y_{2+3 n} y_{3+3 n}\right]-J S^{2}\left[x_{2+3 n} y_{3+3 n}+y_{2+3 n} x_{3+3 n}\right] \\
f_{3+3 n}=-(K+J) S^{2} y_{3+3 n} y_{4+3 n}-\Gamma S^{2}\left[z_{3+3 n} z_{4+3 n}\right. \\
\left.+x_{3+3 n} x_{4+3 n}\right]-J S^{2}\left[z_{3+3 n} x_{4+3 n}+x_{3+3 n} z_{4+3 n}\right]
\end{gathered}
$$

The constraints

$$
x_{j}^{2}+y_{j}^{2}+z_{j}^{2}=1
$$

can be introduced via Lagrange multipliers $\left\{\lambda_{j}\right\}_{j \in \mathbb{Z}}$ so that the free energy becomes

$$
f^{\prime}=f-\frac{1}{2} \sum_{j} \lambda_{j}\left(x_{j}^{2}+y_{j}^{2}+z_{j}^{2}-1\right) .
$$

We will first write down the saddle point equations for a general $J$, and later take $J=0$ in this section.

Seeking classical minima that are invariant under $T_{3 a}$, i.e.,

$$
x_{i+3 n} \equiv x_{i}, y_{i+3 n} \equiv y_{i}, z_{i+3 n} \equiv z_{i}, \quad(1 \leq i \leq 3),
$$

the energy per unit cell $F=3 f^{\prime} / L$ becomes

$$
\begin{aligned}
F= & -\left(K^{\prime}+J^{\prime}\right) x_{1} x_{2}-\Gamma^{\prime}\left(y_{1} y_{2}+z_{1} z_{2}\right)-J^{\prime}\left(y_{1} z_{2}+z_{1} y_{2}\right) \\
& -\left(K^{\prime}+J^{\prime}\right) z_{2} z_{3}-\Gamma^{\prime}\left(x_{2} x_{3}+y_{2} y_{3}\right)-J^{\prime}\left(x_{2} y_{3}+y_{2} x_{3}\right) \\
& -\left(K^{\prime}+J^{\prime}\right) y_{3} y_{1}-\Gamma^{\prime}\left(z_{3} z_{1}+x_{3} x_{1}\right)-J^{\prime}\left(z_{3} x_{1}+x_{3} z_{1}\right) \\
& -\sum_{i=1}^{3} \frac{1}{2} \lambda_{i}\left(x_{i}^{2}+y_{i}^{2}+z_{i}^{2}-1\right)
\end{aligned}
$$

in which $\Gamma^{\prime}, K^{\prime}, J^{\prime}$ are defined as

$$
\Gamma^{\prime}=\Gamma S^{2}, K^{\prime}=K S^{2}, J^{\prime}=J S^{2} .
$$

From Eq. 19, the saddle point equations can be derived as

$$
\begin{aligned}
& \frac{\partial F}{\partial x_{1}}=-\left(K^{\prime}+J^{\prime}\right) x_{2}-\Gamma^{\prime} x_{3}-J^{\prime} z_{3}-\lambda_{1} x_{1}=0 \\
& \frac{\partial F}{\partial y_{1}}=-\Gamma^{\prime} y_{2}-\left(K^{\prime}+J^{\prime}\right) y_{3}-J^{\prime} z_{2}-\lambda_{1} y_{1}=0 \\
& \frac{\partial F}{\partial z_{1}}=-\Gamma^{\prime} z_{2}-\Gamma^{\prime} z_{3}-J^{\prime} x_{3}-J^{\prime} y_{2}-\lambda_{1} z_{1}=0 \\
& \frac{\partial F}{\partial \lambda_{1}}=-\left(x_{1}^{2}+y_{1}^{2}+z_{1}^{2}-1\right)=0 \\
& \frac{\partial F}{\partial x_{2}}=-\left(K^{\prime}+J^{\prime}\right) x_{1}-\Gamma^{\prime} x_{3}-J^{\prime} y_{3}-\lambda_{2} x_{2}=0 \\
& \frac{\partial F}{\partial y_{2}}=-\Gamma^{\prime} y_{1}-\Gamma^{\prime} y_{3}-J^{\prime} z_{1}-J^{\prime} x_{3}-\lambda_{2} y_{2}=0 \\
& \frac{\partial F}{\partial z_{2}}=-\Gamma^{\prime} z_{1}-\left(K^{\prime}+J^{\prime}\right) z_{3}-J^{\prime} y_{1}-\lambda_{2} z_{2}=0 \\
& \frac{\partial F}{\partial \lambda_{2}}=-\left(x_{2}^{2}+y_{2}^{2}+z_{2}^{2}-1\right)=0
\end{aligned}
$$




$$
\begin{aligned}
& \frac{\partial F}{\partial x_{3}}=-\Gamma^{\prime} x_{2}-\Gamma^{\prime} x_{1}-J^{\prime} y_{2}-J^{\prime} z_{1}-\lambda_{3} x_{3}=0 \\
& \frac{\partial F}{\partial y_{3}}=-\Gamma^{\prime} y_{2}-\left(K^{\prime}+J^{\prime}\right) y_{1}-J^{\prime} x_{2}-\lambda_{3} y_{3}=0 \\
& \frac{\partial F}{\partial z_{3}}=-\left(K^{\prime}+J^{\prime}\right) z_{2}-\Gamma^{\prime} z_{1}-J^{\prime} x_{1}-\lambda_{3} z_{3}=0 \\
& \frac{\partial F}{\partial \lambda_{3}}=-\left(x_{3}^{2}+y_{3}^{2}+z_{3}^{2}-1\right)=0 .
\end{aligned}
$$

For the purpose of discussing the Kitaev-Gamma chain in this section, $J$ should be taken as zero. Taking $J=0$, and plugging the following trial solutions

$$
\begin{aligned}
& \hat{n}_{1}^{(0)}=\left(x_{1}, y_{1}, z_{1}\right)^{T}=(a, a, b)^{T}, \\
& \hat{n}_{2}^{(0)}=\left(x_{2}, y_{2}, z_{2}\right)^{T}=(a, b, a)^{T}, \\
& \hat{n}_{3}^{(0)}=\left(x_{3}, y_{3}, z_{3}\right)^{T}=(b, a, a)^{T}, \\
& \lambda_{1}=\lambda_{2}=\lambda_{3}=\lambda,
\end{aligned}
$$

into Eqs. 212223 , where the superscript " $(0)$ " is used to indicate that these are saddle point solutions, we find that Eqs. (21 22 23 are reduced to

$$
\begin{array}{r}
-\left(\lambda+K^{\prime}\right) a-\Gamma^{\prime} b=0 \\
-2 \Gamma^{\prime} a-\lambda b=0 \\
2 a^{2}+b^{2}-1=0 .
\end{array}
$$

Since there are three variables $a, b, \lambda$ and three equations, the solution of Eq. 25 exists. In particular, $\lambda$ can be determined from the secular equation

$$
\operatorname{det}\left(\begin{array}{cc}
-\left(\lambda+K^{\prime}\right) & -\Gamma^{\prime} \\
-2 \Gamma^{\prime} & -\lambda
\end{array}\right)=0 .
$$

When $K=\Gamma$, there are two solutions of $\lambda$ solved from Eq. 26, i.e., $\lambda^{(1)}=\Gamma^{\prime}$ and $\lambda^{(2)}=-2 \Gamma^{\prime}$. The solution $\lambda^{(2)}$ should be kept, since the free energy $F$ in Eq. 19p acquires a larger value for $\lambda^{(1)}$ than for $\lambda^{(2)}$. When $K \neq$ $\Gamma$, Eq. 25 can be solved perturbatively in an expansion over $\Delta$, where the parameter $\Delta$ is defined as

$$
\Delta=(K-\Gamma) / \Gamma
$$

The results up to $O\left(\Delta^{2}\right)$ are

$$
\begin{aligned}
& \lambda(\Delta)=\left(-2-\frac{2}{3} \Delta-\frac{2}{27} \Delta^{2}\right) \Gamma^{\prime}+O\left(\Delta^{3}\right) \\
& a(\Delta)=\frac{1}{\sqrt{3}}\left(1+\frac{1}{9} \Delta-\frac{2}{81} \Delta^{2}\right)+O\left(\Delta^{3}\right) \\
& b(\Delta)=\frac{1}{\sqrt{3}}\left(1-\frac{2}{9} \Delta+\frac{1}{81} \Delta^{2}\right)+O\left(\Delta^{3}\right) .
\end{aligned}
$$

We note that among the two solutions of $\lambda$, the one which reduces to $-2 \Gamma^{\prime}$ for $\Delta=0$ is kept in Eq. 28 .

On the other hand, Eq. 28 only represents a saddle point solution, not necessarily a global minimum of the free energy. Next we perturbatively show that the eigenvalues of the Hessian matrix of the free energy $F$ are all positive at least for $|\Delta| \ll 1$, thereby confirming that Eq. 28 constitutes a minimal solution. Numerics of the classical analysis provide evidence for Eq. (28) to be a global minimum of the free energy as discussed in Appendix B.

Because of the constraints in Eq. 16$]$, the $T_{3 a^{-}}$ invariant spin configurations form a six-dimensional manifold in the nine-dimensional Euclidean space spanned by the nine coordinates $\left\{x_{i}, y_{i}, z_{i}\right\}_{1 \leq i \leq 3}$. Since the $\lambda_{i}$ terms in Eq. (19) vanish as a consequence of the constraints in Eq. (16), $f^{\prime}$ in Eq. (17) acquires the same value as $f$ in Eq. (14) on the six-dimensional manifold, where $L \in 3 \mathbb{Z}$ is the number of lattice sites. Therefore, we will equivalently consider $F=3 f^{\prime} / L$ instead of $3 f / L$ in what follows to calculate the Hessian matrix. The advantage of using $F$ is that its gradient vanishes at the saddle point, unlike the case of $3 f / L$, where the gradient is perpendicular to the tangent space at the saddle point.

Consider the six eigenvalues of the Hessian matrix of the free energy $F$ restricted to the six-dimensional manifold. Right at the FM2 point, two of the eigenvalues are zero, which is reasonable since there are two gapless spin waves for an FM Heisenberg chain. Based on this, we expect that for $|\Delta| \ll 1$, the Hessian matrix contains two low-lying eigenvalues. Since the other four high-lying eigenvalues remain to be gapped with a small correction dependent on $\Delta$, it is enough to check that the two-lying eigenvalues are positive. In what follows, we demonstrate this by perturbatively calculating the two smallest eigenvalues of the Hessian matrix in an expansion in $\Delta$.

Before proceeding on, we first set up some notations. Denote $R(\Delta)=\left(\hat{n}_{1}^{(0), T}(\Delta), \hat{n}_{2}^{(0), T}(\Delta), \hat{n}_{3}^{(0), T}(\Delta)\right)^{T}$ to be the saddle point solution for a fixed value of $\Delta$ within the nine-dimensional space where $\left\{\hat{n}_{i}^{(0)}(\Delta)\right\}_{1 \leq i \leq 3}$ are given by Eqs. 2428. In what follows, we will ignore the transpose operation on the superscripts, bearing in mind that we are always considering a nine-component column vector. Denote $T_{R}(\Delta)$ to be the tangent space of the sixdimensional manifold at the point $R(\Delta)$, and $P(\Delta)$ to be the projection to the tangent space $T_{R}(\Delta)$. Explicitly, the expression of $P(\Delta)$ is

$$
P=\mathbb{1}_{9 \times 9}-r_{1} r_{1}^{T}-r_{2} r_{2}^{T}-r_{3} r_{3}^{T},
$$

in which $r_{i}$ is $r_{i}(\Delta)$ for short, where

$$
\begin{aligned}
& r_{1}=\left(\hat{n}_{1}^{(0)}, \overrightarrow{0}, \overrightarrow{0}\right), \\
& r_{2}=\left(\overrightarrow{0}, \hat{n}_{2}^{(0)}, \overrightarrow{0}\right), \\
& r_{3}=\left(\overrightarrow{0}, \overrightarrow{0}, \hat{n}_{3}^{(0)}\right) .
\end{aligned}
$$

Now let $H_{F}(\Delta)$ be the $9 \times 9$ Hessian matrix of $F$, in which the derivatives are taken with respect to the unconstrained coordinates $\left\{x_{i}, y_{i}, z_{i}\right\}_{1 \leq i \leq 3}$, i.e.,

$$
\left(H_{F}\right)_{\alpha_{i}, \beta_{j}}(\Delta)=\frac{\partial^{2} F}{\partial \alpha_{i} \partial \beta_{j}}(\Delta),
$$

where $1 \leq i, j \leq 3$ are the site indices in a unit cell and $\alpha, \beta=x, y, z$. Notice that if $P(\Delta) H_{F}(\Delta) P(\Delta)$ is 
viewed as a $9 \times 9$ matrix, then there are always three zero eigenvalues, and the three corresponding null vectors are given by Eq. (30), since $r_{i}(\Delta)(1 \leq i \leq 3)$ are always annihilated by $P(\Delta)$. Denote $v_{1}(\Delta), v_{2}(\Delta)$ to be the eigenvectors of the two low-lying eigenvalues, and $w_{1}(\Delta), w_{2}(\Delta), w_{3}(\Delta), w_{4}(\Delta)$ the other four eigenvectors of the high-lying eigenvalues. We will be only interested in $v_{1}(\Delta), v_{2}(\Delta)$.

Consider an FM configuration with all spins aligning along $\hat{n}$-direction. Let $\hat{e}_{\theta}, \hat{e}_{\phi}$ be the two unit vectors perpendicular to $\hat{n}$ which are along tangent directions of the $\theta$ and $\phi$ coodinates, respectively, where $\theta, \phi$ are the polar and azimuthal angles of a unit sphere. When $\Delta=0$, $v_{1}=v_{1}(\Delta=0)$ and $v_{2}=v_{2}(\Delta=0)$ are the two acoustic eigenvectors given by

$$
\begin{aligned}
& v_{1}=\left(\frac{1}{\sqrt{3}} \hat{e}_{\theta}, \frac{1}{\sqrt{3}} \hat{e}_{\theta}, \frac{1}{\sqrt{3}} \hat{e}_{\theta}\right), \\
& v_{2}=\left(\frac{1}{\sqrt{3}} \hat{e}_{\phi}, \frac{1}{\sqrt{3}} \hat{e}_{\phi}, \frac{1}{\sqrt{3}} \hat{e}_{\phi}\right),
\end{aligned}
$$

whereas $\left\{w_{i}=w_{i}(\Delta=0)\right\}_{1 \leq i \leq 4}$ are the optical ones:

$$
\begin{aligned}
& w_{1}=\left(\frac{1}{\sqrt{2}} \hat{e}_{\theta},-\frac{1}{\sqrt{2}} \hat{e}_{\theta}, \overrightarrow{0}\right), \\
& w_{2}=\left(\frac{1}{\sqrt{2}} \hat{e}_{\phi},-\frac{1}{\sqrt{2}} \hat{e}_{\phi}, \overrightarrow{0}\right), \\
& w_{3}=\left(\frac{1}{\sqrt{2}} \hat{e}_{\theta}, \frac{1}{\sqrt{6}} \hat{e}_{\theta},-\sqrt{\frac{2}{3}} \hat{e}_{\theta}\right), \\
& w_{4}=\left(\frac{1}{\sqrt{6}} \hat{e}_{\phi}, \frac{1}{\sqrt{6}} \hat{e}_{\phi},-\sqrt{\frac{2}{3}} \hat{e}_{\phi}\right) .
\end{aligned}
$$

We note that the eigenvalues of the Hessian matrix for $\Delta=0$ corresponding to $v_{i}$-eigenvectors $(i=1,2)$ are both 0 , and those corresponding to $w_{i}$ 's $(i=1,2,3,4)$ are all 3 . Since when $\Delta \rightarrow 0$, the solution reduces to $a=b$ as can be seen from Eq. (28, $\hat{n}$ should be chosen as $\hat{n}_{a}=\frac{1}{3}(1,1,1)^{T}$ to determine the zeroth order terms in $v_{1}(\Delta)$ and $v_{2}(\Delta)$ in a perturbative expansion over $\Delta$. As a result, $\hat{e}_{\theta}$ and $\hat{e}_{\phi}$ in Eqs. (32)33 are given by

$$
\begin{aligned}
& \hat{e}_{\theta}=\left(-\frac{1}{\sqrt{6}},-\frac{1}{\sqrt{6}}, \sqrt{\frac{2}{3}}\right)^{T}, \\
& \hat{e}_{\phi}=\left(-\frac{1}{\sqrt{2}}, \frac{1}{\sqrt{2}}, 0\right)^{T} .
\end{aligned}
$$

The projected Hessian matrix

$$
\mathcal{H}_{F}(\Delta)=P(\Delta) H_{F}(\Delta) P(\Delta)
$$

can be expanded in a power series of $\Delta$

$$
\mathcal{H}_{F}(\Delta)=\mathcal{H}_{F}^{(0)}+\mathcal{H}_{F}^{(1)}+\mathcal{H}_{F}^{(2)}+\ldots
$$

in which $\mathcal{H}_{F}^{(n)}$ is proportional to $\Delta^{n}$. Since both $v_{1}$ and $v_{2}$ have zero eigenvalues of $\mathcal{H}_{F}^{(0)}$, a degenerate first order perturbation theory should be considered, and the first order perturbation Hamiltonian is

$$
h^{(1)}=\left(\begin{array}{ccc}
v_{1}^{T} \mathcal{H}_{F}^{(1)} & v_{1} & v_{1}^{T} \mathcal{H}_{F}^{(1)} v_{2} \\
v_{2}^{T} \mathcal{H}_{F}^{(1)} & v_{1} & v_{2}^{T} \mathcal{H}_{F}^{(1)} v_{2}
\end{array}\right)
$$

However, straightforward calculation shows that $h^{(1)}$ vanishes and we have to go to second order perturbation.

The second order perturbation Hamiltonian can be obtained as

$$
h^{(2)}=\left(\begin{array}{c}
v_{1}^{T} \\
v_{2}^{T}
\end{array}\right)\left(\mathcal{H}_{F}^{(2)}+\mathcal{H}_{F}^{(1)} \sum_{i=1}^{4} \frac{w_{i} w_{i}^{T}}{E_{0}-E_{i}} \mathcal{H}_{F}^{(1)}\right)\left(v_{1} v_{2}\right)(38)
$$

in which $E_{0}=0, E_{i}=3$ are the eigenvalues of $\mathcal{H}_{F}^{(0)}$ corresponding to the acoustic and optical eigenvectors, respectively. Calculations show that

$$
h^{(2)}=\frac{4}{27} \Gamma^{\prime} \Delta^{2} \sigma_{0}
$$

where $\sigma_{0}$ is the $2 \times 2$ identity matrix. Since the eigenvalue $\frac{4}{27} \Gamma S^{2} \Delta^{2}$ is positive, we arrive at the conclusion that the solution in Eq. 24) is indeed a minimum of the classical free energy $F$ regardless of the sign of $\Delta$ at least when $|\Delta|$ is small.

Notice that up to $O\left(\Delta^{2}\right), v_{1}(\Delta)$ and $v_{2}(\Delta)$ are degenerate according to Eq. 39 . In fact, this degeneracy holds to all orders in $\Delta$. This is explained in detail in Appendix C based on a group theory analysis.

\section{B. The symmetry breaking pattern}

To identify the symmetry breaking pattern, we work out the unbroken symmetry group of the spin alignments in Eq. (24) in the six-sublattice rotated frame.

It is straightforward to verify that the spin orientations in Eq. (24) are invariant under the symmetry operations $R_{a} T_{a}$ and $T R_{I} I$. Therefore the unbroken symmetry group $N$ is

$$
N=<R_{a} T_{a}, T R_{I} I>
$$

Since $T_{3 a}$ is unbroken, in what follows within this subsection, we will consider the quotient group $\left.N /<T_{3 a}\right\rangle$. As proved in Ref. 45, $N /\left\langle T_{3 a}\right\rangle$ is isomorphic to $D_{3}$. Here we give a quick demonstration of this isomorphism. The group $D_{n}$ (i.e, the dihedral group of order $2 n$ ) has the following generator-relation representation

$$
D_{n}=<\alpha, \beta \mid \alpha^{n}=\beta^{2}=(\alpha \beta)^{2}=e>.
$$

Define $\alpha=R_{a} T_{a}$, and $\beta=T R_{I} I$. It is straightforward to verify that the relations in Eq. 41) are satisfied for $\alpha, \beta$ modulo $T_{3 a}$. Furthermore, it can be checked that $\left.N /<T_{3 a}\right\rangle$ contains at least six elements. Since $\left|D_{3}\right|=6$, we conclude that $N /\left\langle T_{3 a}\right\rangle \cong D_{3}$. This analysis shows 
that the symmetry breaking pattern predicted by the classical theory is

$$
O_{h} \rightarrow D_{3}
$$

We note that the classical prediction is different from the symmetry breaking pattern for the spin-1/2 case ${ }^{44}$ which is numerically identified as $O_{h} \rightarrow D_{4}$. This indicates strong quantum fluctuations in the spin-1/2 case. On the other hand, numerical calculations provide evidence for the $O_{h} \rightarrow D_{3}$ symmetry breaking for $S=1$ and $S=3 / 2$ as will be discussed in Sec. IIID. Based on this, we conjecture that spin- $1 / 2$ is the only exception and all other spins exhibit an $O_{h} \rightarrow D_{3}$ symmetry breaking as predicted by the classical analysis.

The classical solutions are degenerate, and Eq. 24 only gives one of the possibilities. Since $\left|O_{h}\right|=48$ and $\left|D_{3}\right|=6$, the number of degenerate classical minima is $\left|O_{h} / D_{3}\right|=8$. The other minima are related to Eq. (24) by $O_{h}$ operations. Note that only operations in different equivalent classes of $O_{h} / D_{3}$ give distinct classical spin configurations. In fact, the eight degenerate spin orientations of $\vec{S}_{1}$ are $( \pm a, \pm a, \pm b)$, and the orientations for $\vec{S}_{2}$ and $\vec{S}_{3}$ can be obtained by permuting $a$ and $b$ in accordance with Eq. 24). For a pictorial illustration, the "center of mass" directions of the three spins within a unit cell corresponding to the eight classical minima are represented as solid blue circles located at the vertices of a cube as shown in Fig. 4 .

\section{Spin wave theory}

In this section, we derive the spin wave theory in the path integral formalism which characterizes the small fluctuations around the classical spin configurations. We focus on the $|\Delta| \ll 1$ region, and only the lowest-lying spin wave will be considered.

\section{The spin wave Lagrangian}

The Lagrangin of the spin coherent state path integral is

$$
L=S \sum_{j} \vec{A}_{j} \cdot \partial_{t} \hat{n}_{j}-f^{\prime}\left[\left\{\hat{n}_{j}\right\}\right],
$$

in which the first term is the Berry phase term; the Berry connection $\vec{A}_{j}\left(\theta_{j}, \phi_{j}\right)$ can be chosen as $\frac{1-\cos \theta_{j}}{\sin \theta_{j}} \hat{e}_{j \phi}$ where $\theta_{j}$ and $\phi_{j}$ are the polar and azimuthal angles of $\hat{n}_{j}$, respectively, and $\hat{e}_{j \phi}$ is the unit vector along the azimuthal direction at $\hat{n}_{j}$; the functional $f^{\prime}$ is given by Eq. (17). Notice that again by virtue of the constraints in Eq. (16), there is no difference between $f^{\prime}$ and $f$ in Eq. (14). Therefore, it would be legitimate to write $f^{\prime}$ instead of $f$ in Eq. 43.

Next we expand the Lagrangian around the classical solution in Eq. 24. In the spin wave approximation, only the Gaussian fluctuations will be kept. For small fluctuations, $\hat{n}_{j}$ moves in the tangent space of the unit sphere at the point $\hat{n}_{j}^{(0)}$, in which $\hat{n}_{j}^{(0)}=\hat{n}_{[j]}^{(0)},[j] \equiv j$ $\bmod 3$ and $1 \leq[j] \leq 3$, where $\hat{n}_{[j]}^{(0)}$ is given by Eqs. 2428 . The local coordinate frame of the tangent space at site $j$ can be set up as $\left\{\hat{e}_{\theta}^{(0)}(j), \hat{e}_{\phi}^{(0)}(j)\right\}$, where $\hat{e}_{\theta}^{(0)}(j)$ and $\hat{e}_{\phi}^{(0)}(j)$ are the unit vectors along the polar and azimuthal directions at $\hat{n}_{[j]}^{(0)}$, respectively. Then the deviations away from the equilibrium position are characterized by $\left\{\chi_{\theta}(j), \chi_{\phi}(j)\right\}$ which are the displacements along the $\hat{e}_{\theta}^{(0)}(j)$ and $\hat{e}_{\phi}^{(0)}(j)$ directions.

With the above setup, the Berry phase term becomes

$$
\frac{1}{2} S \sum_{j}\left[\chi_{\theta}(j) \partial_{t} \chi_{\phi}(j)-\chi_{\phi}(j) \partial_{t} \chi_{\theta}(j)\right] .
$$

As can be easily checked, the integration of Eq. 44 over time gives the area swept out by the trajectory of $\hat{n}_{j}$ within the tangent space, which coincides with the geometric meaning of the Berry phase term. We note that $\chi_{\theta}(j)$ and $\chi_{\phi}(j)$ form a pair of canonical conjugates which can be clearly seen from Eq. (44). Alternatively, choosing the quantization axis along $\hat{n}_{j}^{(0)}$, the angular momentum commutation relation becomes

$$
\left[S \chi_{\theta}(j), S \chi_{\phi}(j)\right]=i \hat{n}_{j}^{(0)} \cdot \vec{S}_{j}
$$

Replacing $\hat{n}_{j}^{(0)} \cdot \vec{S}_{j}$ with its classical value $S$, Eq. 45 becomes

$$
\left[\chi_{\theta}(j), \chi_{\phi}(j)\right]=i \frac{1}{S},
$$

which is the canonical commutation relation where $1 / S$ plays the role of $\hbar$. This also indicates that the classical and spin wave analysis only applies in the large- $S$ (i.e., small $\hbar$ ) limit.

For later convenience, we rewrite Eq. 44 in the Cartesian coordinates $\left\{x_{i}, y_{i}, z_{i}\right\}$ in the spin space. The expression under the summation in Eq. (44) can be written as

$$
\hat{n}_{j}^{T}\left[\hat{e}_{\theta}^{(0)}(j) \hat{e}_{\phi}^{(0), T}(j)-\hat{e}_{\phi}^{(0)}(j) \hat{e}_{\theta}^{(0), T}(j)\right] \partial_{t} \hat{n}_{j} .
$$

Notice that the matrix kernel in Eq. 47 is simply the $\pi / 2$-rotation matrix around the $\hat{n}_{j}^{(0)}$-direction. Since such rotation can be implemented by a cross product with $\hat{n}_{j}^{(0)}$, the matrix kernel in Eq. 47 is equal to

$$
M_{j}=\left(\begin{array}{ccc}
0 & -n_{j z}^{(0)} & n_{j y}^{(0)} \\
n_{j z}^{(0)} & 0 & -n_{j x}^{(0)} \\
-n_{j y}^{(0)} & n_{j x}^{(0)} & 0
\end{array}\right),
$$

in which $n_{j \alpha}^{(0)}$ is the $\alpha$-component of $n_{j}^{(0)}$, where $\alpha=$ $x, y, z$ and $j=1,2,3$. Therefore, for small fluctuations, Eq. 43 becomes

$$
L=S \sum_{j} \frac{1}{2} \delta \hat{n}_{j}^{T} M_{j} \partial_{t} \delta \hat{n}_{j}-f^{\prime}\left[\left\{\hat{n}_{j}\right\}\right],
$$


in which $\delta \hat{n}_{j}=\hat{n}_{j}-\hat{n}_{j}^{(0)}$, and $M_{j}$ is given by Eq. 48 .

To discuss the spin wave dispersion, it is convenient to transform into the Fourier space. In what follows, the Fourier transform of the Cartesian coordinates $\alpha_{i+3 n}$ $(\alpha=x, y, z ; i=1,2,3 ; n \in \mathbb{Z})$ will be defined as

$$
\alpha_{i+3 n}=\frac{1}{\sqrt{L / 3}} \sum_{n} e^{i k n} \alpha_{i}(k),
$$

in which $L$ is the system size. Plugging Eq. (50) into Eq. 49) (setting $J=0$ ), we obtain

$$
\begin{aligned}
L= & -f_{0}+S \sum_{k} \frac{1}{2} N^{T}(k) M \partial_{t} N(-k) \\
& -\frac{1}{2} \sum_{k} N^{T}(k)\left[\mathcal{H}_{F}+\delta H(k)\right] N(-k),
\end{aligned}
$$

in which $f_{0}$ is the classical free energy at the saddle points given by

$$
f_{0}=-L \Gamma S^{2}\left(1+\frac{1}{3} \Delta+\frac{1}{27} \Delta^{2}\right)+O\left(\Delta^{3}\right)
$$

$M$ is a $9 \times 9$ matrix

$$
M=\left(\begin{array}{ccc}
M_{1} & 0 & 0 \\
0 & M_{2} & 0 \\
0 & 0 & M_{3}
\end{array}\right),
$$

where $M_{j}(j=1,2,3)$ is defined in Eq. 48,; $N^{T}(k)$ defined as

$$
N^{T}(k)=\left(\hat{n}_{1}^{T}(k), \hat{n}_{2}^{T}(k), \hat{n}_{3}^{T}(k)\right)
$$

is a nine-component row vector where $\hat{n}_{i}^{T}(k)=$ $\left(x_{i}(k), y_{i}(k), z_{i}(k)\right)(i=1,2,3) ; \mathcal{H}_{F}$ is given by Eq. 35; and the $9 \times 9$ matrix $\delta H(k)$ can be derived as

$$
\begin{aligned}
\delta H^{(1,3)}(k) & =\Gamma S^{2}\left(1-e^{-i k}\right) \operatorname{diag}(1,1+\Delta, 1), \\
\delta H^{(3,1)}(k) & =\left[\delta H^{(1,3)}(k)\right]^{\dagger}, \\
\delta H^{(\alpha, \beta)}(k) & =0, \text { for }\{\alpha, \beta\} \neq\{1,3\},
\end{aligned}
$$

where $\operatorname{diag}(\cdot \cdots)$ denotes the diagonal matrix, and $\delta H^{(\alpha, \beta)}(k)$ is the $3 \times 3$ matrix at the $(1,3)$-block of $\delta H(k)$.

\section{Zero wavevector}

Let's first consider the zero wavevector spin waves. The Hamiltonian in Eq. 51 for $k=0$ is

$$
\frac{1}{2} N^{T}(k=0) H_{F} N(k=0) \text {. }
$$

To get the spin wave masses, the matrix kernel in Eq. (56) needs to be diagonalized. Naively, the matrix $\mathcal{H}_{F}$ has already been diagonalized in Sec. III A. However, in Eq. (56), $\mathcal{H}_{F}$ must be diagonalized by symplectic transformation which leaves the symplectic form $M$ in
Eq. 53 invariant, unlike the case in Sec. III A where $\mathcal{H}_{F}$ is diagonalized by an orthogonal transformation. Recall that we have already proved $\mathcal{H}_{F}$ to be positive definite which is the restriction of $H_{F}$ in Eq. (31) to the six-dimensional tangent space. Then by the symplectic theory $^{\text {? }}, \mathcal{H}_{F}$ (which is viewed as a $6 \times 6$ matrix) can be diagonalized by a symplectic transformation $U$, i.e.,

$$
\mathcal{H}_{F}=U^{T} \Lambda U
$$

in which $U$ satisfies

$$
U^{T} M U=M
$$

and the diagonal matrix $\Lambda$ is of the form

$$
\Lambda=\left(\begin{array}{ccc}
m_{1} \sigma_{0} & 0 & 0 \\
0 & m_{2} \sigma_{0} & 0 \\
0 & 0 & m_{3} \sigma_{0}
\end{array}\right)
$$

where $m_{3}>m_{2}>m_{1}>0(i=1,2,3) ; \sigma_{0}$ is the $2 \times 2$ identity matrix; and $M$ is viewed as a $6 \times 6$ matrix acting in the six-dimensional tangent space. We will be interested in $m_{1}$ which is related to the smallest spin wave mass. Notice that in general, $m_{i}$ 's do not coincide with the eigenvalues of $\mathcal{H}_{F}$. In what follows, $m_{1}$ will be calcualted to the leading nonvanishing order in $\Delta$ by perturbation theory. The result happens to be the same as the two lowest eigenvalues of $\mathcal{H}_{F}$ (i.e., $\frac{4}{27} \Gamma S^{2} \Delta^{2}$ ) derived in Sec. ПIA.

The calculations of $m_{i}$ 's can be converted into an eigenvalue problem by considering the matrix $M \mathcal{H}_{F}{ }^{\text {? }}$. In fact, according to the symplectic linear algebra, the eigenvalues of $M \mathcal{H}_{F}$ are $\pm i m_{j}(j=1,2,3)$. The basics of the symplectic transformations for our purpose are collected in Appendix D. In what follows, we will view both $M$ and $\mathcal{H}_{F}$ as $9 \times 9$ matrices. Since $M_{j}$ is defined as a cross product operation in Eq. $48, \hat{n}_{j}^{(0)}$ must be a null vector of $M_{j}$. As a result, $r_{i}(\Delta)$ in Eq. (30) are always annihilated by $M$. Hence the $9 \times 9$ matrix $M \mathcal{H}_{F}$ always has three zero eigenvalues, and we will be interested in the other six eigenvalues. When $\Delta=0$, among the other six eigenvalues, $M(\Delta=0) \mathcal{H}_{F}(\Delta=0)$ contains two zero eigenvalues with eigenvectors given by

$$
v_{ \pm}=v_{1} \pm i v_{2}
$$

where $v_{i}=v_{i}(\Delta=0)(i=1,2)$ are given by Eq. (32). For $\Delta \neq 0, v_{ \pm}$evolve into $v_{ \pm}(\Delta)$ which have eigenvalues $\pm \operatorname{im}_{1}(\Delta)$.

Let's first consider $\pm i m_{1}(\Delta)$ to the linear order of $\Delta$. Define $\mathcal{H}_{F}^{M,(n)}$ in terms of the power expansion as

$$
M(\Delta) \mathcal{H}_{F}(\Delta)=\mathcal{H}_{F}^{M,(0)}+\mathcal{H}_{F}^{M,(1)}+\mathcal{H}_{F}^{M,(2)}+\ldots
$$

where $\mathcal{H}_{F}^{M,(n)}$ is proportional to $\Delta^{n}$. Define $P_{1}(\Delta)$ to be projection to the subspace spanned by $v_{ \pm}(\Delta)$. At a nonzero $\Delta$, the first order degenerate perturbation theory is given by

$$
h^{M,(1)}=P_{1}^{(0)} \mathcal{H}_{F}^{M,(1)} P_{1}^{(0)},
$$


where $P_{1}^{(0)}=P_{1}(\Delta=0)$. Calculations show that $h^{M,(1)}$ vanishes, hence second order degenerate perturbation has to be considered. We note that there is a quick way to see $h^{M,(1)}=0$. In fact, there is the relation

$$
h^{M,(1)}=M^{(0)} P_{1}^{(0)} h^{(1)} P_{1}^{(0)},
$$

in which $h^{(1)}$ is defined in Eq. (37). A proof of Eq. 63 is given in Appendix E Since $P_{1}^{(0)} h^{(1)} P_{1}^{(0)}$ vanishes according to the discussion below Eq. (37), $h^{M,(1)}$ has to vanish as a result.

Next we proceed to second order perturbation. Define $u_{i}(i=1,2,3,4)$ as

$$
\begin{aligned}
& u_{1}=w_{1}+i w_{2}, \\
& u_{2}=w_{1}-i w_{2}, \\
& u_{3}=w_{3}+i w_{4}, \\
& u_{4}=w_{3}-i w_{4},
\end{aligned}
$$

in which $w_{i}$ are given in Eq. (33). Then $u_{i}$ are eigenvectors of $\mathcal{H}_{F}^{M,(0)}$ with eigenvalues equal to $\epsilon_{i}$, where

$$
\epsilon_{1}=3 i, \epsilon_{2}=-3 i, \epsilon_{3}=3 i, \epsilon_{4}=-3 i \text {. }
$$

The second order degenerate perturbation theory is captured by the following matrix,

$$
\begin{aligned}
h^{M,(2)}=\left(\begin{array}{c}
v_{+}^{\dagger} \\
v_{-}^{\dagger}
\end{array}\right)\left[\mathcal{H}_{F}^{M,(2)}+\mathcal{H}_{F}^{M,(1)} \sum_{i=1}^{4}\right. & \left.\frac{u_{i} u_{i}^{\dagger}}{E_{0}-\epsilon_{i}} \mathcal{H}_{F}^{M,(1)}\right] \\
& \times\left(v_{+} v_{-}\right),
\end{aligned}
$$

in which $E_{0}=0$ are the eigenvalues of $\mathcal{H}_{F}^{M,(0)}$ for $v_{ \pm}$. Calculations show that

$$
h^{M,(2)}=i \frac{4}{27} \Gamma^{\prime} \Delta^{2} \sigma_{3},
$$

in which $\sigma_{3}$ is the third Pauli matrix. This shows that to the leading nonvanishing order,

$$
m_{1}=\frac{4}{27} \Gamma^{\prime} \Delta^{2} .
$$

We have numerically calculated the eigenvalues of $M(\Delta) \mathcal{H}_{F}(\Delta)$ and the result for $m_{1}$ is displayed in Fig. 5 . As can be seen from Fig. 5, the numerical results agree well with Eq. (68).

\section{Nonzero wavevectors and the spin wave dispersions}

Next, we consider nonzero wavevectors and diagonalize the matrix kernel $H_{F}+\delta H(k)$ in Eq. (51). We will consider the long wavelength limit $k \ll 1$ where the lattice constant has been taken as 1 . As can be seen from Eq. (55), the matrix elements of $\delta H(k)$ are very small in the long wavelength limit, hence $\delta H(k)$ can be treated as a perturbation of $\mathcal{H}_{F}$.

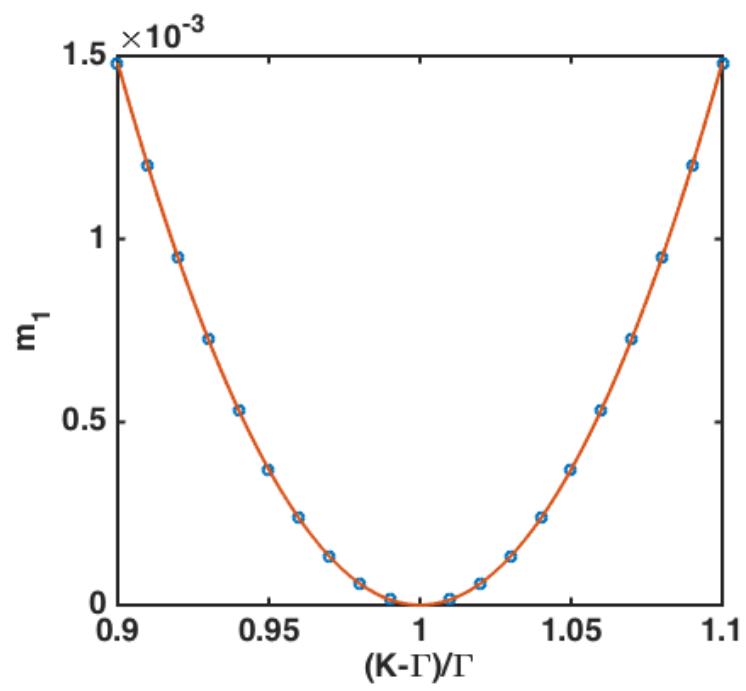

FIG. $5: m_{1}$ vs. $\Delta$ for $J=0$ represented by the hollow circles as obtained by numerical diagonalization of $M(\Delta) \mathcal{H}_{F}(\Delta)$, where $\Delta$ is defined in Eq. 27). The solid line represents $\frac{4}{27} \Delta^{2}$. The vertical axis is in unit of $\Gamma^{\prime}=\Gamma S^{2}$.

Multiplying with the symplectic matrix, the first order degenerate perturbation is implemented by the following $2 \times 2$ matrix

$$
\delta h^{(1)}(k)=P_{1}^{(0)} M^{(0)} \delta H(\Delta=0, k) P_{1}^{(0)},
$$

in which we have taken $\Delta=0$ since we are only interested in the leading nonvanishing order terms in $\Delta$. Straightforward calculations show that the eigenvalues of $\delta h^{(1)}(k)$ are $\pm i \frac{1}{6} \Gamma S^{2} k^{2}$. Thus, by keeping only the lowest-lying spin wave, the spin wave Lagrangian in Eq. (51) becomes

$$
\begin{aligned}
& L=-f_{0}+S \sum_{k} \frac{1}{2}\left(\xi_{\theta}(k) \partial_{t} \xi_{\phi}(-k)-\xi_{\phi}(k) \partial_{t} \xi_{\theta}(-k)\right) \\
& -\frac{1}{2} \Gamma S^{2} \sum_{k}\left(\frac{4}{27} \Delta^{2}+\frac{1}{3} k^{2}\right)\left[\xi_{\theta}(k) \xi_{\theta}(-k)+\xi_{\phi}(k) \xi_{\phi}(-k)\right],
\end{aligned}
$$

in which

$$
\xi_{\theta}(k)=N^{T}(k) v_{1}, \xi_{\phi}(k)=N^{T}(k) v_{2},
$$

where $N(k)$ is defined in Eq. (54).

Finally, we rewrite the spin wave Hamiltonian (i.e., the second line in Eq. (70) in real space in the continuum limit. The summation over $k$ can be converted to $\sum_{n}=\frac{1}{3} \int d x$ where $x$ is the real space coordinate in the continuum limit. The momentum $k$ can be converted to $i \partial_{n}=3 i \partial_{x}$. Using these, we see that the spin wave Hamiltonian $H_{s w}$ in the real space is

$$
H_{s w}=\Gamma S^{2} \int d x\left[\frac{1}{2}\left(\partial_{x} \xi_{\theta}\right)^{2}+\frac{1}{2}\left(\partial_{x} \xi_{\phi}\right)^{2}+\frac{2}{81} \Delta^{2}\left(\xi_{\theta}^{2}+\xi_{\phi}^{2}\right)\right],
$$




\begin{tabular}{|c||c||c|c|c|}
\hline$E(S=1)$ & No field & $h_{\hat{x}}=10^{-4}$ & $h_{\hat{n}_{a}}=10^{-4}$ & $h_{\hat{n}_{I}}=10^{-4}$ \\
\hline$E_{1}$ & -12.01911 & -12.02010 & -12.02067 & -12.02106 \\
$E_{2}-E_{1}$ & $1.57 \cdot 10^{-4}$ & $1.47 \cdot 10^{-4}$ & $1.17 \cdot 10^{-3}$ & $2.3 \cdot 10^{-4}$ \\
$E_{3}-E_{1}$ & $1.57 \cdot 10^{-4}$ & $2.21 \cdot 10^{-4}$ & $1.17 \cdot 10^{-3}$ & $2.06 \cdot 10^{-3}$ \\
$E_{4}-E_{1}$ & $1.57 \cdot 10^{-4}$ & $4.07 \cdot 10^{-4}$ & $1.21 \cdot 10^{-3}$ & $2.06 \cdot 10^{-3}$ \\
$E_{5}-E_{1}$ & $3.38 \cdot 10^{-4}$ & $2.09 \cdot 10^{-3}$ & $2.36 \cdot 10^{-3}$ & $2.22 \cdot 10^{-3}$ \\
$E_{6}-E_{1}$ & $3.38 \cdot 10^{-4}$ & $2.21 \cdot 10^{-3}$ & $2.40 \cdot 10^{-3}$ & $2.25 \cdot 10^{-3}$ \\
$E_{7}-E_{1}$ & $3.38 \cdot 10^{-4}$ & $2.28 \cdot 10^{-3}$ & $2.40 \cdot 10^{-3}$ & $4.16 \cdot 10^{-3}$ \\
$E_{8}-E_{1}$ & $5.55 \cdot 10^{-4}$ & $2.41 \cdot 10^{-3}$ & $3.60 \cdot 10^{-3}$ & $4.30 \cdot 10^{-3}$ \\
$E_{9}-E_{1}$ & $4.33 \cdot 10^{-3}$ & $4.34 \cdot 10^{-3}$ & $4.60 \cdot 10^{-3}$ & $4.50 \cdot 10^{-3}$ \\
$E_{10}-E_{1}$ & $4.33 \cdot 10^{-3}$ & $4.48 \cdot 10^{-3}$ & $5.56 \cdot 10^{-3}$ & $5.32 \cdot 10^{-3}$ \\
\hline
\end{tabular}

\begin{tabular}{|c|c||c|c|c|}
\hline$E(S=3 / 2)$ & No field & $h_{\hat{x}}=10^{-4}$ & $h_{\hat{n}_{a}}=10^{-4}$ & $h_{\hat{n}_{I}}=10^{-4}$ \\
\hline$E_{1}$ & -26.99084 & -26.99237 & -26.99342 & -26.99389 \\
$E_{2}-E_{1}$ & $6.6 \cdot 10^{-5}$ & $6.3 \cdot 10^{-5}$ & $1.78 \cdot 10^{-3}$ & $8.2 \cdot 10^{-5}$ \\
$E_{3}-E_{1}$ & $6.6 \cdot 10^{-5}$ & $8.2 \cdot 10^{-5}$ & $1.78 \cdot 10^{-3}$ & $3.09 \cdot 10^{-3}$ \\
$E_{4}-E_{1}$ & $6.6 \cdot 10^{-5}$ & $1.48 \cdot 10^{-4}$ & $1.78 \cdot 10^{-3}$ & $3.09 \cdot 10^{-3}$ \\
$E_{5}-E_{1}$ & $1.34 \cdot 10^{-4}$ & $3.11 \cdot 10^{-3}$ & $3.57 \cdot 10^{-3}$ & $3.15 \cdot 10^{-3}$ \\
$E_{6}-E_{1}$ & $1.34 \cdot 10^{-4}$ & $3.16 \cdot 10^{-3}$ & $3.57 \cdot 10^{-3}$ & $3.15 \cdot 10^{-3}$ \\
$E_{7}-E_{1}$ & $1.34 \cdot 10^{-4}$ & $3.18 \cdot 10^{-3}$ & $3.57 \cdot 10^{-3}$ & $6.21 \cdot 10^{-3}$ \\
$E_{8}-E_{1}$ & $2.04 \cdot 10^{-4}$ & $3.23 \cdot 10^{-3}$ & $1.27 \cdot 10^{-3}$ & $6.26 \cdot 10^{-3}$ \\
$E_{9}-E_{1}$ & $1.00 \cdot 10^{-2}$ & $1.01 \cdot 10^{-2}$ & $1.21 \cdot 10^{-2}$ & $1.02 \cdot 10^{-2}$ \\
$E_{10}-E_{1}$ & $1.00 \cdot 10^{-2}$ & $1.01 \cdot 10^{-2}$ & $5.37 \cdot 10^{-2}$ & $1.10 \cdot 10^{-2}$ \\
\hline
\end{tabular}

TABLE I: Energies of the first ten lowest lying states computed with DMRG. The data refer to $L=18$ sites, $J=0$, and $\phi=0.2 \pi$. The energies enclosed by the colored squares are approximately degenerate.

in which $\xi_{\theta}(j), \xi_{\phi}(j)$ is a pair of canonical conjugates satisfying $\left[\xi_{\theta}(j), \xi_{\phi}\left(j^{\prime}\right)\right]=i \delta_{j j^{\prime}} \frac{1}{S}$. From Eq. 72 and the fact that $\hbar=1 / S$, the dispersion of the spin wave can be obtained as

$$
E(k)=\Gamma S\left(k^{2}+\frac{4}{81} \Delta^{2}\right) .
$$

Since the spin wave mass $\frac{4}{81} \Gamma S \Delta^{2}$ is very small, it would be very difficult to determine numerically (for example, in DMRG numerics). We note that the path integral calculations to derive the spin wave Hamiltonian in Eq. 72 . is equivalent with the Bogoliubov transformation based on the Holstein-Primakoff transformation as explained in detail in Appendix F.

\section{DMRG numerics}

In this section, we present DMRG numerical results for $S=1,3 / 2$, which provide evidence for the revealed $O_{h} \rightarrow$ $D_{3}$ symmetry breaking based on a classical analysis.

Table I displays the results for the energies of the ten lowest eigenstates under different magnetic fields at a representative point $(J=0, \phi=0.2 \pi)$ in the $O_{h} \rightarrow D_{3}$ phase, in which the first and second tables are for $S=1$ and $S=3 / 2$, respectively. DMRG is performed on a system of $L=18$ sites in obtaining the data. As can be clearly seen from Table I], the system is approximately eight-fold degenerate at zero field, with a ground state energy splitting (characterized by $E_{8}-E_{1}$ ) about one order of magnitude smaller than the excitation gap $E_{9}-E_{1}$, which is consistent with the eight-fold degeneracy predicted by the $O_{h} \rightarrow D_{3}$ symmetry breaking as discussed in Sec. IIIB.

To test the pattern of the spin alignments as shown in Fig. 4. we apply small magnetic fields $h_{\hat{x}}, h_{\hat{n}_{a}}$ and $h_{\hat{n}_{I}}$ along $\hat{x}, \hat{n}_{a}$, and $\hat{n}_{I}$-directions (within the six-sublattice rotated frame), respectively, where $\hat{n}_{a}$ and $\hat{n}_{I}$ are defined in Eq. (8). The magnitude of the field $h=10^{-4}$ is chosen to satisfy $\Delta E \ll S|h| L \ll E_{g}$, in which $L$ is the system size, $E_{g}$ is the excitation gap, and $\Delta E$ is the finite size splitting of the ground state octet at zero field. Such choice of $h$ ensures a degenerate perturbation within the eight-dimensional ground state subspace, and at the same time, no mixing between the ground states and the excited states is induced. Hence, it is a thermodynamically small field which only perturbs the ground state subspace.

As can be read from Fig. 4, the field $h_{\hat{x}}$ is predicted to lower the energies of the four states located at vertices $(1, \pm 1, \pm 1) ; h_{\hat{n}_{a}}$ lowers the energy of state at $(1,1,1)$; and $h_{\hat{n}_{I}}$ lowers the energies of the two states at $(1, \pm 1,-1)$. Indeed, as can be seen from Table [ the ground state degeneracy becomes 4-, 1- and 2-fold under the fields $h_{\hat{x}}$, $h_{\hat{n}_{a}}$, and $h_{\hat{n}_{I}}$, respectively, which are consistent with the above analysis. This provides further evidence for the predicted $O_{h} \rightarrow D_{3}$ symmetry breaking.

In addition, we have also directly measured the expectation values of the spin operators under the fields $h_{\hat{n}_{a}}$ and $h_{\hat{n}_{b}}$ where $\hat{n}_{b}$ is along the $(1,1,-1)$-direction. The results are displayed in Figs. (6 7). According to the discussions in Secs. (III A, III B), since the vertices located at $(1,1,1)$ and $(1,1,-1)$ are picked out by $h_{\hat{n}_{a}}$ and $h_{\hat{n}_{b}}$, respectively, the spin alignments are predicted to be:

$$
\begin{aligned}
& \left\langle\vec{S}_{1+3 n}\right\rangle=(a, a, b)^{T}, \\
& \left\langle\vec{S}_{3+3 n}\right\rangle=(a, b, a)^{T}, \\
& \left\langle\vec{S}_{3+3 n}\right\rangle=(b, a, a)^{T},
\end{aligned}
$$

for $h_{\hat{n}_{a}} ;$ and

$$
\begin{aligned}
& \left\langle\vec{S}_{1+3 n}\right\rangle=(a, a,-b)^{T}, \\
& \left\langle\vec{S}_{2+3 n}\right\rangle=(a, b,-a)^{T}, \\
& \left\langle\vec{S}_{3+3 n}\right\rangle=(b, a,-a)^{T},
\end{aligned}
$$

for $h_{\hat{n}_{b}}$. Indeed, Fig. 6 (Fig. 7) is consistent with the pattern in Eq. (74) (Eq. (75)).

\section{THE NÉEL PHASE FOR $J>0$}

In this section, we perform a combination of classical and spin wave analysis for $J>0$ in the vicinity of the 


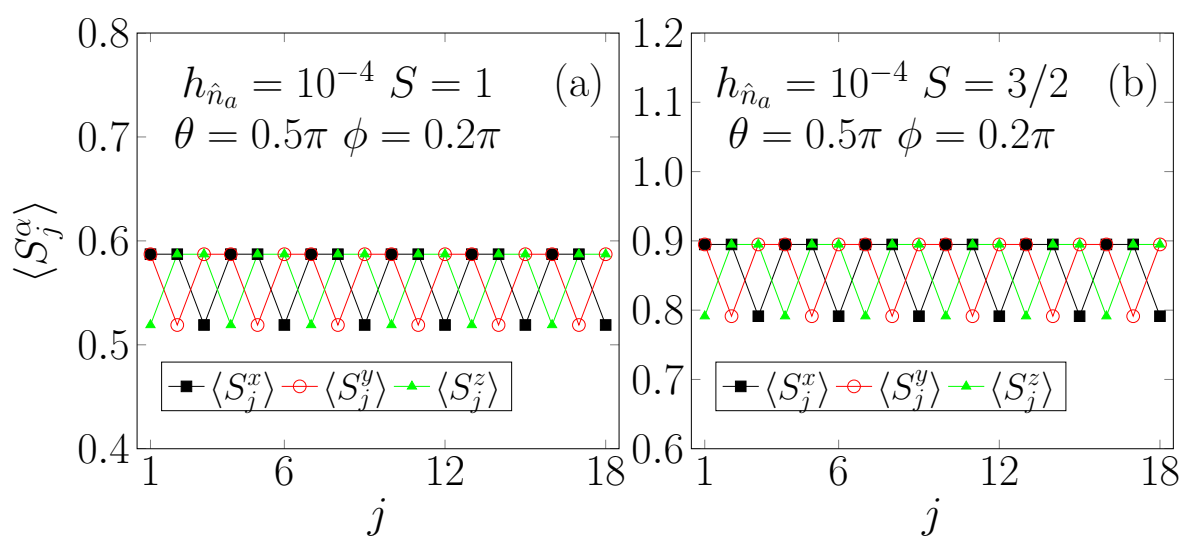

FIG. 6: Spin expectation values $\left\langle S_{i}^{\alpha}\right\rangle(\alpha=x, y, z)$ under a small field $h_{\hat{n}_{a}}=10^{-4}$ along $(1,1,1)$-direction at a representative point $(\theta=\pi / 2, \phi=0.2 \pi)$ in the $O_{h} \rightarrow D_{3}$ phase for (a) $S=1$, and (b) $S=3 / 2$. The parametrization $(\theta, \phi)$ is defined in Eq. (4). DMRG simulations are performed on a system of $L=18$ sites.

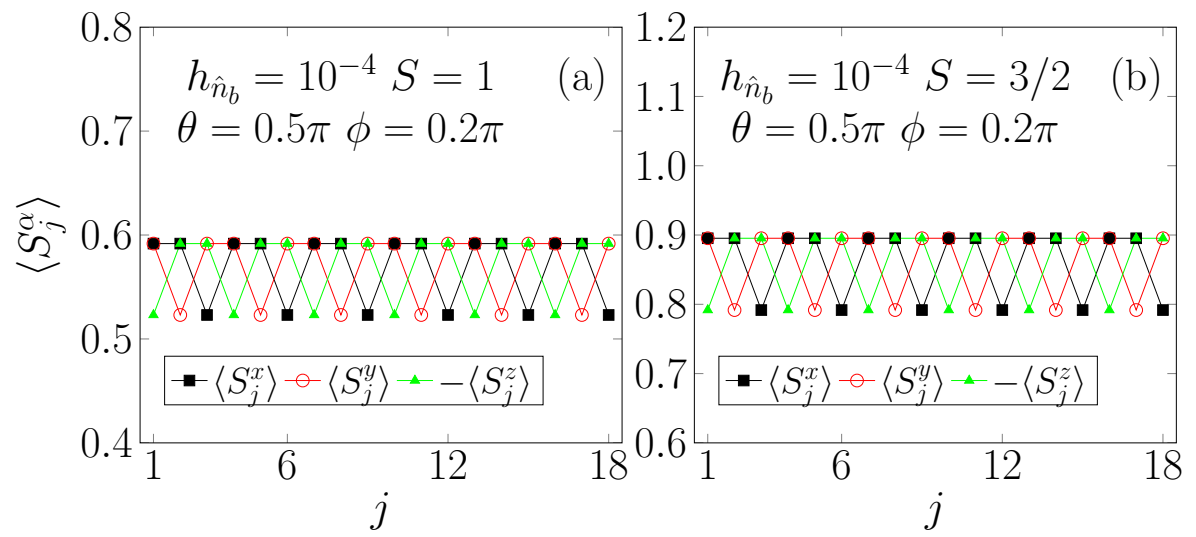

FIG. 7: Spin expectation values $\left\langle S_{i}^{\alpha}\right\rangle(\alpha=x, y, z)$ under a small field $h_{\hat{n}_{b}}=10^{-4}$ along $(1,1,-1)$-direction at a representative point $(\theta=\pi / 2, \phi=0.2 \pi)$ in the $O_{h} \rightarrow D_{3}$ phase for (a) $S=1$, and (b) $S=3 / 2$. The parametrization $(\theta, \phi)$ is defined in Eq. (4). DMRG simulations are performed on a system of $L=18$ sites.

FM2 point in Fig. 1] Since the spin alignments exhibit an antiferromagnetic pattern in the original frame, the region corresponds to a Néel phase. The mass of the lowest spin wave is calculated to the leading nonvanishing order in an expansion over $J$ and $\Delta$. Throughout this section, we work in the six-sublattice rotated frame unless otherwise stated.

\section{A. Classical analysis and spin wave theory}

The saddle point equations have been derived in Eqs. (21)22 23). Assuming the same pattern of spin alignments and relations between $\lambda_{i}$ 's $(1 \leq i \leq 3)$ as those in Eq. (24), the saddle point equations reduce to

$$
\begin{aligned}
-\left(\lambda+K^{\prime}+2 J^{\prime}\right) a-\Gamma^{\prime} b & =0 \\
-2 \Gamma^{\prime} a-\left(\lambda+2 J^{\prime}\right) b & =0 \\
2 a^{2}+b^{2}-1 & =0
\end{aligned}
$$

Since there are three variables and three equations, a solution in general exists. On the other hand, to confirm that this is a minimum of the free energy, we still need to show that the eigenvalues of the Hessian matrix are all positive. We will do a perturbative analysis and demonstrate that this is true at least in the vicinity of the FM2 point.

For simplicity, let's first take $\Delta=0$ and turn on a small $J>0$. The solution of Eq. 76 is given by

$$
\lambda=-2 \Gamma^{\prime}-2 J^{\prime}, a=b=\frac{1}{\sqrt{3}} .
$$

Following the same logic in Sec. IIIA, we define the matrix

$$
\mathcal{H}_{F}(J)=P(J) H_{F}(J) P(J),
$$

in which $H_{F}(J)$ is the $9 \times 9$ Hessian matrix of the free energy in Eq. 190 , and $P(J) \equiv P(J=0)$ is given by Eq. 29 where $\vec{r}_{i}=\hat{n}_{a}(i=1,2,3)$ with $\hat{n}_{a}=\frac{1}{\sqrt{3}}(1,1,1)^{T}$. Taking the two gapless acoustic eigenvectors $v_{1}$ and $v_{2}$ 
(defined in Eq. $(32)$ ) as the zeroth order vectors, the first order degenerate perturbation matrix is given by

$$
h^{(1)}(J)=\left(\begin{array}{c}
v_{1}^{T} \\
v_{2}^{T}
\end{array}\right) \Delta \mathcal{H}_{F}(J)\left(v_{1} v_{2}\right),
$$

in which

$$
\Delta \mathcal{H}_{F}(J)=\mathcal{H}_{F}(\Delta=0, J)-\mathcal{H}_{F}(\Delta=0, J=0) .
$$

Straightforward calculations show that

$$
h^{(1)}(J)=2 J S^{2} \sigma_{0},
$$

where $\sigma_{0}$ is the $2 \times 2$ identity matrix. Thus the eigenvalues of the Hessian matrix are positive when $J>0$, thereby confirming the solution in Eq. (77) to be at least a local minimum. In fact, numerical minimization of the free energy shows that it is also a global minimum as discussed in Appendix B.

We note that the above analysis can be extended to the case where both $J$ and $\Delta$ are nonzero but small (i.e., $J,|\Delta| \ll 1$ ). To the lowest nonvanishing order in perturbation, the wavefunction is unchanged. Hence the eigenvalues are additive for $J$ and $\Delta$. Therefore, two lowest eigenvalues of the Hessian matrix are both $2 J S^{2}+\frac{4}{27} \Delta^{2} \Gamma S^{2}$.

We also briefly discuss the symmetry breaking in the Néel phase. The unbroken symmetry group is the same as the $O_{h} \rightarrow D_{3}$ phase, since the spins have the same pattern of alignments. As discussed in Sec. IIB, the full symmetry group for a nonzero $J$ is $D_{3 d}$ (modulo $T_{3 a}$ ), therefore, the symmetry breaking in the Néel phase is

$$
D_{3 d} \rightarrow D_{3} .
$$

Since $\left|D_{3 d} / D_{3}\right|=2$, there are two degenerate ground states. The "center of mass" directions for the three spins within a unit cell in the two degenerate states are plotted as the two solid light blue circles in Fig. 4.

We make a comment on the spin ordering in the original frame. Rotating the spin orientations in Eq. 24) back to the original frame using Eq. (5), it is straightforward to verify that the spins align in a Néel pattern with a two-site periodicity, i.e.,

$$
\vec{S}_{1+2 n}=S(a, a, b)^{T}, \vec{S}_{2+2 n}=S(-a,-a,-b)^{T} .
$$

Thus this phase is termed as "Néel" in the phase diagram in Fig. 1.

Finally we build up a spin wave theory for the small fluctuations around the classical configurations. To obtain the spin wave mass, we need to calculate the eigenvalues of the matrix $M(\Delta, J) \mathcal{H}_{F}(\Delta, J)$. The contribution from the $\Delta$-part is the same as Sec. IIIC, For the $J$-part, within first order perturbation theory, the contribution is the same as the eigenvalues of $\mathcal{H}_{F}$ as can be seen from Eq. 63. Therefore, the spin wave Hamiltonian for the lowest spin wave is

$$
\begin{aligned}
H_{s w}= & \frac{1}{2} \Gamma S^{2} \int d x\left[\left(\partial_{x} \xi_{\theta}\right)^{2}+\left(\partial_{x} \xi_{\phi}\right)^{2}\right] \\
& +\left(\frac{2}{81} \Gamma \Delta^{2}+\frac{1}{3} J\right) S^{2} \int d x\left(\xi_{\theta}^{2}+\xi_{\phi}^{2}\right),
\end{aligned}
$$

\begin{tabular}{|c|c|c|}
\hline$E(S=1)$ & No field & $h_{\hat{n}_{a}}=10^{-4}$ \\
\hline$E_{1}$ & -17.96054 & -17.96348 \\
$E_{2}-E_{1}$ & $1.57 \cdot 10^{-6}$ & $5.887 \cdot 10^{-3}$ \\
$E_{3}-E_{1}$ & $6.054 \cdot 10^{-2}$ & $6.056 \cdot 10^{-2}$ \\
$E_{4}-E_{1}$ & $6.054 \cdot 10^{-2}$ & $6.110 \cdot 10^{-2}$ \\
$E_{5}-E_{1}$ & $6.515 \cdot 10^{-2}$ & $6.518 \cdot 10^{-2}$ \\
\hline
\end{tabular}

\begin{tabular}{|c|c|c|}
\hline$E(S=3 / 2)$ & No field & $h_{\hat{n}_{a}}=10^{-4}$ \\
\hline$E_{1}$ & -39.50646 & -39.51099 \\
$E_{2}-E_{1}$ & $\underline{3.9 \cdot 10^{-12}}$ & $9.060 \cdot 10^{-3}$ \\
$E_{3}-E_{1}$ & $8.908 \cdot 10^{-2}$ & $8.910 \cdot 10^{-2}$ \\
$E_{4}-E_{1}$ & $8.908 \cdot 10^{-2}$ & $8.997 \cdot 10^{-2}$ \\
$E_{5}-E_{1}$ & $9.487 \cdot 10^{-2}$ & $9.489 \cdot 10^{-2}$ \\
\hline
\end{tabular}

TABLE II: Energies of the five lowest lying states computed with DMRG simulations. The data refer to $L=18$ sites, $\theta=0.4 \pi$, and $\phi=0.2 \pi$. The energies enclosed by the colored squares are approximately degenerate.

in which $\xi_{\theta}(j), \xi_{\phi}(j)$ is a pair of canonical conjugates satisfying $\left[\xi_{\theta}(j), \xi_{\phi}\left(j^{\prime}\right)\right]=i \delta_{j j^{\prime}} \frac{1}{S}$.

\section{B. DMRG numerics}

In this section, we present DMRG numerical results for $S=1,3 / 2$, which provide evidence for the revealed $D_{3 d} \rightarrow D_{3}$ symmetry breaking based on a classical analysis. We proceed similarly as Sec. IIID.

Table II displays the results for the energies of the five lowest eigenstates under different magnetic fields at a representative point $(\theta=0.4 \pi, \phi=0.2 \pi)$ in the Néel phase, in which the first and second tables are for $S=1$ and $S=3 / 2$, respectively, and $\theta, \phi$ are defined in Eq. (4). DMRG is performed for a system of $L=18$ sites in obtaining the data. On a $L=12$ size system, we have checked that the DMRG results are in agreement with Lanczos Exact Diagonalization. As can be clearly seen from Table II, the system is approximately two-fold degenerate at zero field, with a ground state energy splitting (characterized by $E_{2}-E_{1}$ ) orders of magnitude smaller than the excitation gap $E_{3}-E_{1}$, which is consistent with the two-fold degeneracy predicted by the $D_{3 d} \rightarrow D_{3}$ symmetry breaking as discussed in Eq. 82. We have also applied a small magnetic field along the $\hat{n}_{a}$-direction, which should be able to pick out the state located at the (111)-vertex as shown in Fig. 4. Indeed, as can be seen from Table II the system becomes nondegenerate when $h_{\hat{n}_{a}}$ is applied.

In addition, we have also directly measured the expectation values of the spin operators under the fields $h_{\hat{n}_{a}}$. The results are displayed in Fig. 8 (a) for $S=1$ and (b) for $3 / 2$. Clearly, the spin alignments revealed in Fig. 8 are consistent with the pattern in Eq. (74). 


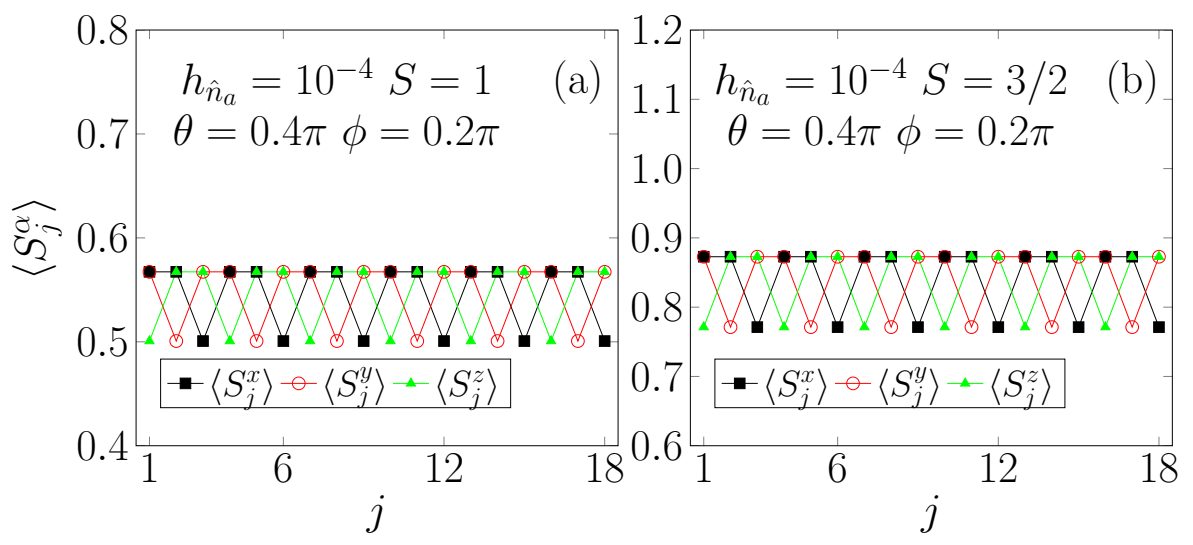

FIG. 8: Spin expectation values $\left\langle S_{i}^{\alpha}\right\rangle(\alpha=x, y, z)$ under a small field $h_{\hat{n}_{a}}=10^{-4}$ along $(1,1,1)$-direction at a representative point $(\theta=0.4 \pi, \phi=0.2 \pi)$ in the Néel phase for (a) $S=1$, and (b) $S=3 / 2$. The parametrization $(\theta, \phi)$ is defined in Eq. (4). DMRG is performed on a system of $L=18$ sites.

\section{THE " $D_{3}$-BREAKING I, II" PHASES FOR $J<0$}

In this section, we discuss the " $D_{3}$-breaking I, II" phases in the negative $J$ region. We work within the six-sublattice rotated frame unless otherwise stated.

\section{A. Classical phase diagram}

We first briefly describe the classical phase diagram in the negative $J$ region as shown in Fig. 1, with calculations included in the next two subsections. There are two phases denoted as " $D_{3}$ breaking I" and " $D_{3}$ breaking II". Both phases break the $D_{3}$ symmetry albeit in different ways, hence the ground states are six-fold degenerate. However, the symmetry breaking patterns are not the same.

To clarify this point, recall that the symmetry group is $G_{1} \simeq D_{3 d} \ltimes 3 \mathbb{Z}$ as discussed in Sec. IIB since $T_{3 a}$ is not broken, we consider $G_{1}^{\prime}=G_{1} /\left\langle T_{3 a}>\simeq D_{3 d}\right.$ and the spins within a unit cell in what follows. In the " $D_{3}$ breaking I" phase, the spin orientations in one of the six degenerate ground states are

$$
\vec{S}_{1}=S\left(\begin{array}{l}
x \\
y \\
z
\end{array}\right), \vec{S}_{2}=S\left(\begin{array}{c}
-\frac{1}{\sqrt{2}} \\
0 \\
\frac{1}{\sqrt{2}}
\end{array}\right), \vec{S}_{3}=S\left(\begin{array}{l}
-z \\
-y \\
-x
\end{array}\right)
$$

in which $x^{2}+y^{2}+z^{2}=1$. As can be checked, the little group of Eq. (85) is generated by $R_{I} I$. Hence the symmetry breaking is $D_{3 d} \rightarrow<R_{I} I>$. On the other hand, in the " $D_{3}$ breaking II" phase, the spin orientations in one of the six degenerate ground states are

$$
\vec{S}_{1}=S\left(\begin{array}{c}
x \\
y \\
z
\end{array}\right), \vec{S}_{2}=S\left(\begin{array}{c}
m \\
n \\
m
\end{array}\right), \vec{S}_{3}=S\left(\begin{array}{c}
z \\
y \\
x
\end{array}\right),
$$

in which $x^{2}+y^{2}+z^{2}=2 m^{2}+n^{2}=1$. The little group of Eq. 860 is generated by $T R_{I} I$, and the symmetry breaking is $D_{3 d} \rightarrow\left\langle T R_{I} I>\right.$. Thus we see that although the symmetry breaking in the two phases are both $D_{3 d} \rightarrow$ $\mathbb{Z}_{2}$, the group $\mathbb{Z}_{2}$ represents different little groups. We also note that since $D_{3 d} / \mathbb{Z}_{2} \simeq D_{3}$, the two phases both exhibit $D_{3}$-breaking which is the origin of the names of the two phases. The "center of mass" directions of the three spins within a unit cell in the six degenerate ground states are shown in Fig. 4, where the red (dark blue) solid circles correspond to the " $D_{3}$-breaking I (II)" phases.

\section{B. The " $D_{3}$ breaking I" phase}

\section{The classical solution}

We perform a classical analysis in the " $D_{3}$ breaking I" phase. For simplicity, we consider the $\Delta=0$ case with a small negative $J$. We will use the normalized parameter $\bar{J}=J / \Gamma$.

We take the trial solution given by Eq. (85) and assume $\lambda_{3}=\lambda_{1}$. Setting $K=\Gamma$ and plugging the trial solution into Eq. $21|22| 23)$, the saddle point equations reduce to

$$
\begin{aligned}
\left(-\lambda_{1}+J^{\prime}\right) x+\Gamma^{\prime} z+\frac{1}{\sqrt{2}}\left(K^{\prime}+J^{\prime}\right) & =0 \\
\left(\Gamma^{\prime}+J^{\prime}-\lambda_{1}\right) y-\frac{1}{\sqrt{2}} J^{\prime} & =0 \\
\Gamma^{\prime} x+\left(J^{\prime}-\lambda_{1}\right) z-\frac{1}{\sqrt{2}} \Gamma^{\prime} & =0 \\
-\left(K^{\prime}+J^{\prime}\right) x+J^{\prime} y+\Gamma^{\prime} z+\frac{1}{\sqrt{2}} \lambda_{2} & =0 \\
x^{2}+y^{2}+z^{2}-1 & =0 .
\end{aligned}
$$

Since there are five variables $x, y, z, \lambda_{1}, \lambda_{2}$ and five equations, generically a solution exists. Eq. (87) can be solved 
perturbatively in an expansion over $J$. The results up to $O\left(J^{3}\right)$ are

$$
\begin{aligned}
& x=-\frac{1}{\sqrt{2}}-\frac{1}{6 \sqrt{2}} \bar{J}+\frac{5}{72 \sqrt{2}} \bar{J}^{2}-\frac{7}{432 \sqrt{2}} \bar{J}^{3}+O\left(\bar{J}^{4}\right), \\
& y=\frac{1}{3 \sqrt{2}} \bar{J}-\frac{1}{18 \sqrt{2}} \bar{J}^{2}+\frac{1}{216 \sqrt{2}} \bar{J}^{3}+O\left(\bar{J}^{4}\right), \\
& z=\frac{1}{\sqrt{2}}-\frac{1}{6 \sqrt{2}} \bar{J}-\frac{1}{72 \sqrt{2}} \bar{J}^{2}+\frac{5}{432 \sqrt{2}} \bar{J}^{3}+O\left(\bar{J}^{4}\right),
\end{aligned}
$$

and

$$
\begin{aligned}
& \lambda_{1}=\Gamma^{\prime}\left(-2+\frac{1}{2} \bar{J}-\frac{1}{24 \sqrt{2}} \bar{J}^{2}-\frac{1}{144} \bar{J}^{3}\right)+O\left(\bar{J}^{4}\right), \\
& \lambda_{2}=\Gamma^{\prime}\left(-2-\bar{J}-\frac{5}{12} \bar{J}^{2}+\frac{7}{72} \bar{J}^{3}\right)+O\left(\bar{J}^{4}\right) .
\end{aligned}
$$

Detailed derivations of Eqs. 88 are included in Appendix $\mathrm{G}$.

Consider the projected Hessian matrix defined in Eq. (78) for $J<0$. The perturbation Hamiltonian is

$$
\begin{aligned}
\Delta \mathcal{H}_{F}(J)= & P(J) H_{F}(J) P(J) \\
& -P(J=0) H_{F}(J=0) P(J=0) .
\end{aligned}
$$

Then the first order degenerate perturbation Hamiltonian is given by

$$
h^{(1)}(J)=\left(\begin{array}{cc}
v_{1}^{T} \Delta \mathcal{H}_{F}(J) v_{1} & v_{1}^{T} \Delta \mathcal{H}_{F}(J) v_{2} \\
v_{2}^{T} \Delta \mathcal{H}_{F}(J) v_{1} & v_{2}^{T} \Delta \mathcal{H}_{F}(J) v_{2}
\end{array}\right)
$$

in which $v_{1}, v_{2}$ are given by Eq. 32 where

$$
\hat{e}_{\theta}=\left(-\frac{1}{\sqrt{2}}, 0,-\frac{1}{\sqrt{2}}\right)^{T}, \hat{e}_{\phi}=(0,-1,0)^{T} .
$$

Calculations show that

$$
h^{(1)}(J)=-J S^{2}\left(\begin{array}{cc}
\frac{4}{3} & \frac{2 \sqrt{2}}{3} \\
\frac{2 \sqrt{2}}{3} & \frac{2}{3}
\end{array}\right) .
$$

The two eigenvalues of $h^{(1)}(J)$ are 0 and $-2 J S^{2}$. Thus we see that although the first order perturbation already breaks the degeneracy, one eigenvalue remains zero up to $O(J)$ and higher order perturbation is needed to obtain a nonzero value. In fact, calculations show that the first nonvanishing term for this eigenvalue appears at $O\left(J^{3}\right)$. Here we only mention that the result is $-\frac{1}{2} \Gamma S^{2} \bar{J}^{3}$, and detailed derivations are given in Appendix $G$

In summary, the two low-lying eigenvalues are

$$
-\Gamma S^{2} \bar{J},-\frac{1}{2} \Gamma S^{2} \bar{J}^{3}
$$

which are both positive when $J<0$. This shows that Eqs. (88 89) represent a minimum of the free energy. Numerical calculations provide evidence for Eqs. 88 89p to be a global minimum as discussed in Appendix B

\section{Spin wave theory}

In this subsection, we calculate the lowest-lying spin wave mass for the $\Delta=0$ case with a small negative $J$. Let's first consider the case of a zero wavevector. Again, we need to diagonalize the Hessian matrix $\mathcal{H}_{F}(J)$ using symplectic transformations. As discussed in Sec. III C 2 the spin wave masses are given by the eigenvalues of $M(J) \mathcal{H}_{F}(J)$. We will calculate the smallest spin wave mass up to the leading nonvanishing order of $J$.

Before proceeding on, notice that the definitions of $v_{i}$ $(i=1,2), w_{j}(j=1,2,3,4)$ are the same as Eq. (32) and Eq. 33), where $\hat{e}_{\theta}$ and $\hat{e}_{\phi}$ should be taken as Eq. (92). We emphasize that we will use the same notations as Sec. III C 2 for simplicity. However, the expressions of the quantities are different from those in Eq. (III C 2), which are determined by the form of the Hamiltonian and the saddle point solutions. Let $P_{1}^{(0)}$ be the projection operation to the subspace spanned by $\left\{v_{1}, v_{2}\right\}$. Let $\mathcal{H}^{M,(n)}(J)$ be the order $J^{n}$ term in the expansion of $M(J) \mathcal{H}_{F}(J)$ over $J$. Then the first order degenerate perturbation is given by the following $2 \times 2$ matrix,

$$
h^{M,(1)}(J)=P_{1}^{(0)} \mathcal{H}_{F}^{M,(1)}(J) P_{1}^{(0)} .
$$

According to Eq. (63), this is simply

$$
h^{M,(1)}(J)=i \sigma_{2} h^{(1)}(J),
$$

in which $h^{(1)}(J)$ is given by Eq. 93 , and $i \sigma_{2}$ is the projection of $M^{(0)}$ to the subspace spanned by $\left\{v_{1}, v_{2}\right\}$ where $\sigma_{\alpha}(\alpha=1,2,3)$ are the Pauli matrices. As can be readily checked, since one of the two eigenvalues of $h^{(1)}(J)$ vanishes, the two eigenvalues of $h^{M,(1)}(J)$ are both zero. Hence, we need to go to second order perturbation.

The second order perturbation is given by the following matrix

$$
\begin{array}{r}
h^{M,(2)}(J)=\left(\begin{array}{c}
v_{1}^{T} \\
v_{2}^{T}
\end{array}\right)\left[\mathcal{H}_{F}^{M,(2)}+\mathcal{H}_{F}^{M,(1)} \sum_{i=1}^{4} \frac{u_{i} u_{i}^{\dagger}}{E_{0}-\epsilon_{i}} \mathcal{H}_{F}^{M,(1)}\right] \\
\times\left(v_{1} v_{2}\right),
\end{array}
$$

in which $v_{ \pm}$and $u_{i}(i=1,2,3,4)$ are defined in the same way as Eq. 60 and Eq. 64); and the eigenvalues $\epsilon_{i}$ 's $(i=1,2,3,4)$ are given by

$$
\epsilon_{1}=-3 i, \epsilon_{2}=3 i, \epsilon_{3}=-3 i, \epsilon_{4}=3 i .
$$

Calculations show that

$$
h^{M,(2)}(J)=0 \text {. }
$$

Hence, the second order perturbation also vanishes, which means that we have to go to the third order perturbation theory.

The third order perturbation matrix $h^{M,(3)}(J)$ is given by

$$
h^{M,(3)}(J)=\bar{J}^{3}\left(\begin{array}{cc}
-\frac{19}{54 \sqrt{2}} & \frac{35}{108} \\
-\frac{4}{27} & \frac{19}{54 \sqrt{2}}
\end{array}\right) .
$$



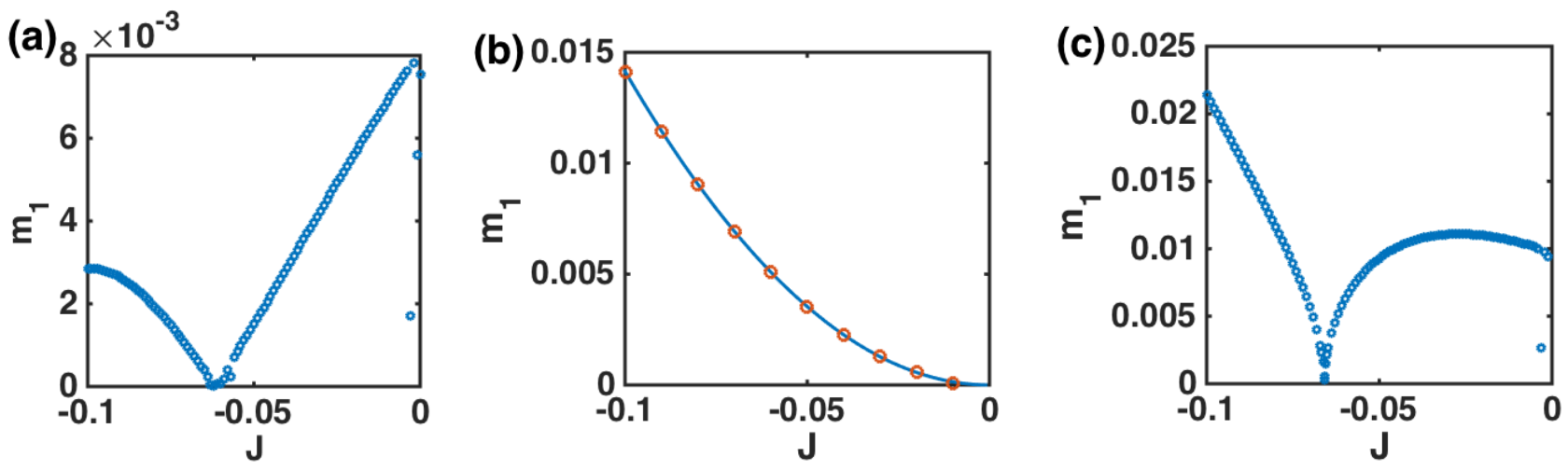

FIG. 9: Numerically obtained smallest spin wave mass $m_{1}$ vs. $J$ represented by the hollow circles for (a) $\varphi=0.21 \pi,($ b) $\varphi=0.25 \pi$, and (c) $\varphi=0.30 \pi$. In all figures, $m_{1}$ is in units of $\Gamma S^{2}$, where $\Gamma=\sin (\varphi)$. In (b), the solid line represents $\Gamma^{\prime} \bar{J}^{2}$ where $\Gamma^{\prime}=\frac{1}{\sqrt{2}}$.

Detailed derivation of Eq. 100 is included in Appendix G3.

The eigenvalues of $h^{M,(1)}+h^{M,(2)}+h^{M,(3)}$ are $\pm i \Gamma S^{2} \bar{J}^{2}$, which gives $m_{1}(\Delta=0, J)=\Gamma S^{2} \bar{J}^{2}$. In Fig. 9 (b), the hollow circles represent the numerical results for $m_{1}$ by numerically solving the eigenvalues of $M(J) \mathcal{H}_{F}(J)$, and the solid line represents $\Gamma^{\prime} \bar{J}^{2}$. As can be clearly seen, the numerical results agree well with the obtained perturbative results up to $O\left(J^{2}\right)$.

Based on the above discussions, we are able to obtain the spin wave Hamiltonian for $\Delta=0,|\bar{J}| \ll 1$ as

$$
\begin{aligned}
H_{s w}= & \frac{1}{2} \Gamma S^{2} \int d x\left[\left(\partial_{x} \xi_{\theta}\right)^{2}+\left(\partial_{x} \xi_{\phi}\right)^{2}\right] \\
& +\frac{1}{6} \Gamma S^{2} \bar{J}^{2} \int d x\left(\xi_{\theta}^{2}+\xi_{\phi}^{2}\right) .
\end{aligned}
$$

\section{The " $D_{3}$ breaking II" phase}

In this subsection, we discuss the " $D_{3}$ breaking II" phase. To obtain an intuitive understanding, let's start with the case of $\Delta \neq 0, J=0$, and then turn on a small negative $J$. At $J=0$, the symmetry breaking is $O_{h} \rightarrow D_{3}$ and there are eight degenerate ground states. If $J \neq 0$, since $J$ is planar-like, the two states along $\pm(111)$-direction in Fig. 4 will have higher energies than the other six states. As a result, the two solid light blue circles at the $\pm(111)$ vertices should be removed compared with the $J=0$ case as shown in Fig. 4 . Thus, the ground states now are six-fold degenerate and the spin orientations are slightly distorted away from the $J=0$ case. This is different from the " $D_{3}$ breaking I" phase where the "center of mass" direction is perpendicular to the (111)-direction. On the other hand, when $J$ is large enough, the "center of mass" spin orientations will eventually be bent to the plane perpendicular to the (111)direction. Thus we expect a " $D_{3}$ breaking II" to " $D_{3}$ breaking I" phase transition classically, this is indeed the case as shown in Fig. 1.
We take the trial solution in Eq. 86 and assume $\lambda_{3}=$ $\lambda_{1}$. Under these assumptions, Eqs. 2122223 reduce to

$$
\begin{aligned}
-\left(J^{\prime}+\lambda_{1}\right) x-\Gamma^{\prime} z-\left(K^{\prime}+J^{\prime}\right) m & =0 \\
-\left(K^{\prime}+J^{\prime}+\lambda_{1}\right) y-J^{\prime} m-\Gamma^{\prime} n & =0 \\
-\Gamma^{\prime} x-\left(J^{\prime}+\lambda_{1}\right) z-\Gamma^{\prime} m-J^{\prime} n & =0 \\
-\left(K^{\prime}+J^{\prime}\right) x-J^{\prime} y-\Gamma^{\prime} z-\lambda_{2} m & =0 \\
-2 \Gamma^{\prime} y-2 J^{\prime} z-\lambda_{2} n & =0 \\
x^{2}+y^{2}+z^{2}-1 & =0 \\
2 m^{2}+n^{2}-1 & =0 .
\end{aligned}
$$

Since there are seven variables and seven equations, generically a solution exists.

Next we try to solve Eq. 102 in a perturbative expansion over $J$. However, we find difficulty in carrying out a perturbative expansion. The $J=0$ case has been already solved in Sec. IIIA, which is taken as the zeroth order solution. When $J \neq 0$, up to $O(J)$, the solution is (see Appendix $\mathrm{H}$ for details)

$$
\begin{aligned}
x & =\frac{1}{\sqrt{3}}\left[x_{0}+\left(\frac{3}{\Delta^{2}}+\frac{7}{6 \Delta}\right) \bar{J}\right]+O\left(\bar{J}^{2}\right) \\
y & =\frac{1}{\sqrt{3}}\left[-x_{0}+\left(\frac{6}{\Delta^{2}}+\frac{1}{3 \Delta}\right) \bar{J}\right]+O\left(\bar{J}^{2}\right) \\
z & =\frac{1}{\sqrt{3}}\left[z_{0}+\left(\frac{3}{\Delta^{2}}+\frac{1}{6 \Delta}\right) \bar{J}\right]+O\left(\bar{J}^{2}\right), \\
m & =\frac{1}{\sqrt{3}}\left[x_{0}+\left(\frac{3}{\Delta^{2}}-\frac{5}{6 \Delta}\right) \bar{J}\right]+O\left(\bar{J}^{2}\right) \\
n & =\frac{1}{\sqrt{3}}\left[-z_{0}+\left(\frac{6}{\Delta^{2}}+\frac{1}{3 \Delta}\right) \bar{J}+O\left(\bar{J}^{2}\right)\right],
\end{aligned}
$$

and

$$
\begin{aligned}
& \lambda_{1}=\lambda_{0}+\frac{2}{\Delta} \bar{J}+O\left(\bar{J}^{2}\right) \\
& \lambda_{2}=\lambda_{0}-\frac{4}{\Delta} \bar{J}+O\left(\bar{J}^{2}\right),
\end{aligned}
$$



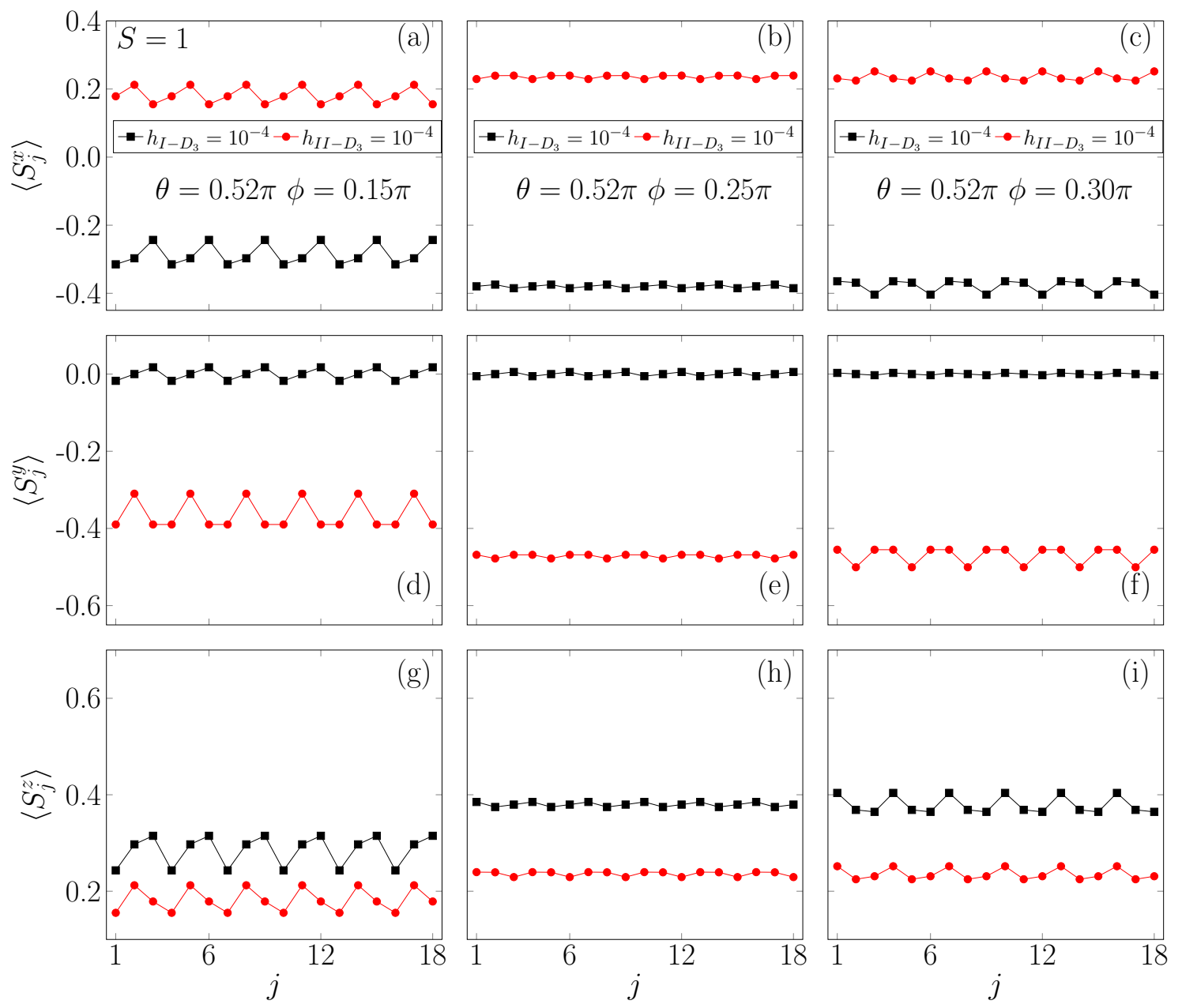

FIG. 10: (a,b,c) $\left\langle S_{j}^{x}\right\rangle,(\mathrm{d}, \mathrm{e}, \mathrm{f})\left\langle S_{j}^{y}\right\rangle$, and $(\mathrm{g}, \mathrm{h}, \mathrm{i})\left\langle S_{j}^{z}\right\rangle$ vs $j$ under $h_{\mathrm{I}}$ (black squares) and $h_{\mathrm{II}}$ (red dots) fields for $S=1$ at several different points. (a,d,g) are for $(\theta=0.52 \pi, \phi=0.15 \pi)$; (b,e,h) for $(\theta=0.52 \pi, \phi=0.25 \pi)$; and $(\mathrm{c}, \mathrm{f}, \mathrm{i})$ for $(\theta=0.52 \pi, \phi=0.30 \pi)$. DMRG numerics are performed on $L=18$ sites with periodic boundary conditions. Both $h_{\mathrm{I}}$ and $h_{\mathrm{II}}$ fields are taken to be $10^{-4}$.

in which the results are obtained up to $O(J)$. In particular, since the $J$-dependent terms contain negative powers of $\Delta$, the perturbation is valid only when $|\bar{J}| / \Delta^{2} \ll 1$.

As usual, the eigenvalues of the Hessian matrix should be calculated to verify that the saddle point solution in Eqs. 103 104 105 corresponds to a minimum of the free energy. However, the nonanalyticity in $\Delta$ in Eqs. 103 104 105 complicates the calculation. As discussed in detail in Appendix $\mathrm{H}$, one possibly has to go up to at least fifth order perturbation in $\Delta$. We will not perform such a difficult fifth order perturbation, and in fact, we suspect if a good perturbation exists because of the nonanalytical dependence of the saddle point solution on $\Delta$. The smallest eigenvalue is studied by numerics as discussed in Appendix $\mathrm{H}$.

Due to the above mentioned difficulty, the spin wave mass will not be perturbatively calculated. Instead, we study the spin wave mass numerically by computing the eigenvalues of the matrix $M(\Delta, J) \mathcal{H}_{F}(\Delta, J)$. The dependence of $m_{1}$ on $J$ at three representatively values of $\varphi=0.21 \pi$ and $0.30 \pi$ are shown in Fig. 9 (a) and (c), respectively. The value of $J=J_{c}(\varphi)$ where $m_{1}$ vanishes is the transition point between the " $D_{3}$-breaking I" and the " $D_{3}$-breaking II" phases. Notice that for fixed value of $\varphi$, the " $D_{3}$-breaking I (II)" phase occupies the region $J>J_{c}(\varphi)\left(J<J_{c}(\varphi)\right)$.

\section{DMRG numerics}

In this subsection, we present the DMRG numerical results which provide numerical evidence for the predicted " $D_{3}$-breaking I, II" phases for both $S=1$ and $3 / 2$.

Before proceeding on, we mention a subtlety in numerical calculations, which has already been discussed in detail in Ref. 46, In either the " $D_{3}$-breaking I" or " $D_{3}$ breaking II" phases, the six symmetry breaking ground states only become exactly degenerate in the thermodynamic limit. In a finite size system, the ground state can be some arbitrary linear combination of the six states, and the coefficients depend on the system size and numerical details. Because of this, random cancellations occur 

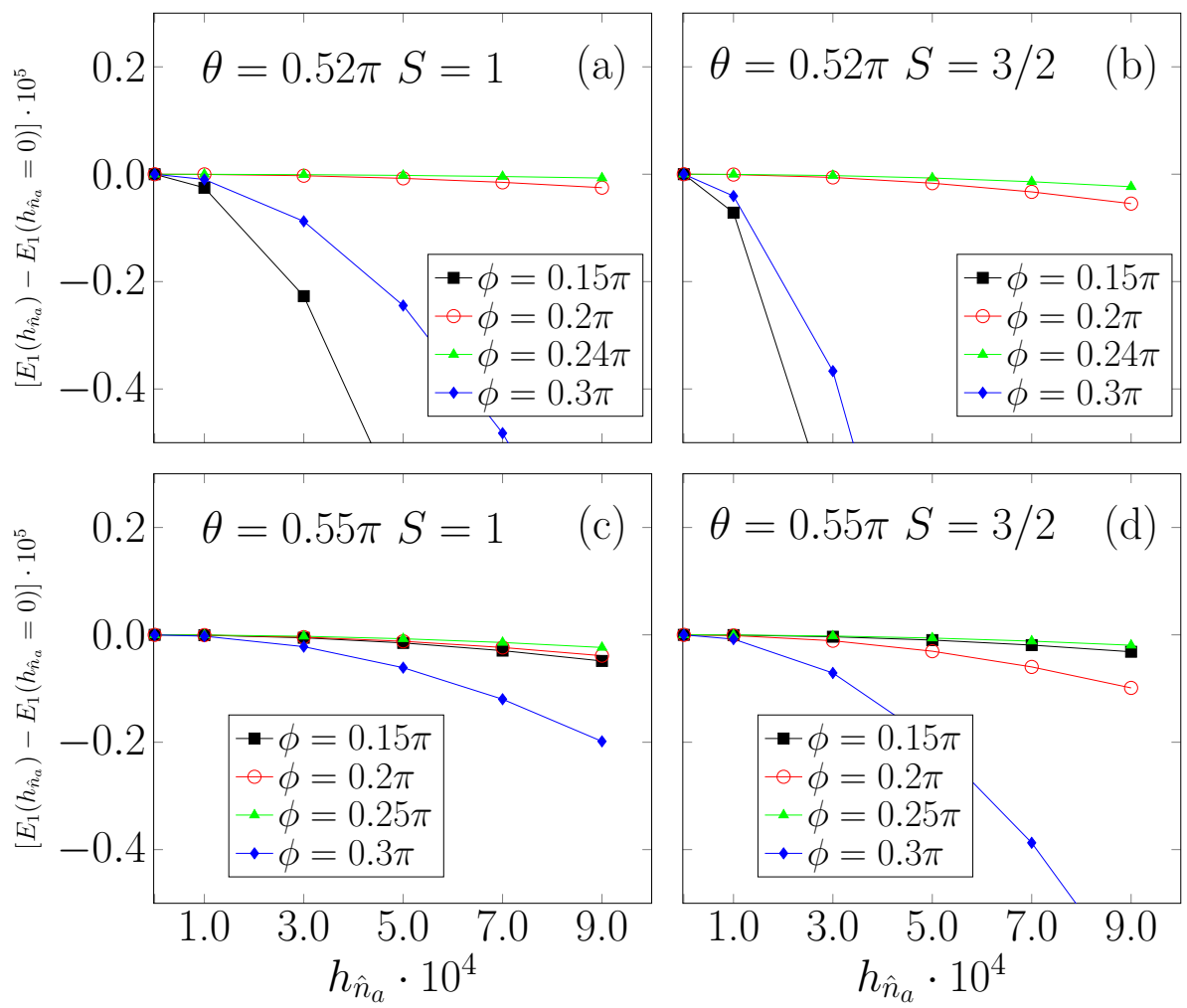

FIG. 11: $\Delta E\left(=E\left(h_{\hat{n}_{a}}\right)-E\left(h_{\hat{n}_{a}}=0\right)\right)$ vs $h_{\hat{n}_{a}}$ for $(\mathrm{a}, \mathrm{c}) S=1$, and (b,d) $S=3 / 2$ at fixed values of $\theta$ and $\varphi$. The magnetic field $h_{\hat{n}_{a}}$ is taken along the (111)-direction with a magnitude $h_{\hat{n}_{a}}=5 \times 10^{-4}$. ED numerics are performed on $L=18$ sites with periodic boundary conditions.

if the correlation functions $\left\langle S_{i}^{\alpha} S_{i+r}^{\beta}\right\rangle$ or the expectation values of the spin operators $\left\langle\vec{S}_{i}\right\rangle$ are directly computed. To circumvent such difficulty, a small magnetic field has to be applied such that the system is polarized into one of the six degenerate ground states.

For our purpose, we choose the field to be $h_{\mathrm{I}}$ along the $(-1,0,1)$-direction in the " $D_{3}$-breaking I" phase, and $h_{\mathrm{II}}$ along the $(1,-1,1)$-direction in the " $D_{3}$-breaking II" phase. According to Fig. 4, we expect that the red solid circle located at $(-1,0,1)$ is picked out in the the " $D_{3^{-}}$ breaking I" phase, and the solid dark blue circle located at $(1,-1,1)$ is picked out in the the " $D_{3}$-breaking II" phase. Then with the application of such fields, the spins should align according to the pattern given in Eq. (85) (Eq. (86)) in the " $D_{3}$-breaking I (II)" phase. However, as discussed in Ref. 46, the " $D_{3}$-breaking I (II)" phase responds to the $h_{\mathrm{II}^{-}}\left(h_{(\mathrm{I})^{-}}\right)$field as does the " $D_{3}$-breaking II (I)" phase. Therefore, this method is not able to distinguish the two $D_{3}$-breaking phases. However, the method is still useful since it can test the existence of either " $D_{3}$ breaking I" or " $D_{3}$-breaking II" orders.

We have calculated the spin expectation values $\left\langle S_{j}^{\alpha}\right\rangle$ $(\alpha=x, y, z)$ at three representative points $(\theta=$ $0.52 \pi, \phi=0.15 \pi),(\theta=0.52 \pi, \phi=0.25 \pi)$ and $(\theta=$ $0.52 \pi, \phi=0.30 \pi)$ under the $h_{\mathrm{I}}$ and $h_{\mathrm{II}}$ fields. The results for $S=1$ are displayed in Fig. 10. DMRG numerics are performed on a system of $L=18$ sites with periodic boundary conditions, and both $h_{\mathrm{I}}$ and $h_{\mathrm{II}}$ fields are taken to be $10^{-4}$. As can be clearly seen from Fig. 10. the spin alignments are consistent with the patterns given in Eqs. (85 86), thereby confirming the existence of the " $D_{3}$-breaking I, II" phases. We have also studied the $S=3 / 2$ case, and the results are included in Appendix I.

As discussed in Ref. 46, the two $D_{3}$-breaking phases can be distinguished by studying the response of the system to a small field $h_{\hat{n}_{a}}$ along the (111)-direction, since the " $D_{3}$-breaking I" phase does not respond to $h_{\hat{n}_{a}}$, whereas the " $D_{3}$-breaking II" phase does have an response.

Fig. 11 shows the energy change $\Delta E=E\left(h_{\hat{n}_{a}}\right)-$ $E\left(h_{\hat{n}_{a}}=0\right)$ as a function of $h_{\hat{n}_{a}}$ at several representative points in the negative $J$ region for both $S=1$ and $S=3 / 2$. Clearly, while the system has a huge response at some points, the response nearly vanishes at others. Based on the results in Fig. 11, we arrive at the conclusion that the points $(\theta=0.52 \pi, \phi=0.2 \pi, 0.24 \pi)$ and $(\theta=0.55 \pi, \phi=0.15 \pi, 0.2 \pi, 0.25 \pi)$ are within the " $D_{3}$ breaking I" phase, whereas the points $(\theta=0.52 \pi, \phi=$ $0.15 \pi, 0.3 \pi)$ and $(\theta=0.55 \pi, \phi=0.3 \pi)$ are in the " $D_{3}$ breaking II" phase. In particular, as can be seen from Fig. 11, the range of the " $D_{3}$-breaking I" phase expands by increasing $\theta$, which is consistent with the classical phase diagram as shown in Fig. 1 . 

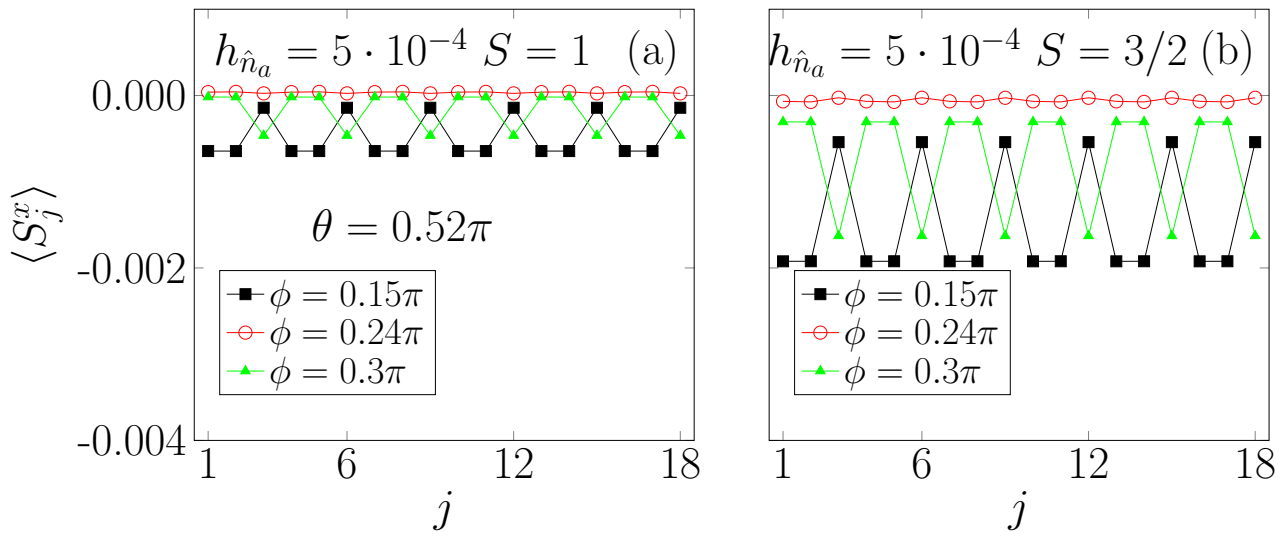

FIG. 12: $\left\langle S_{j}^{x}\right\rangle$ vs $j$ for (a) $S=1$, and (b) $S=3 / 2$ at fixed values of $\theta, \varphi$. The magnetic field is taken along the (111)-direction with a magnitude $h_{\hat{n}_{a}}=5 \times 10^{-4}$. DMRG numerics are performed on $L=18$ sites with periodic boundary conditions.

Fig. 12 displays the response of $\left\langle S_{j}^{x}\right\rangle$ to $h_{\hat{n}_{a}}=5 \times 10^{-4}$ at several different points for both $S=1$ and $S=3 / 2$. As can be seen from Fig. 12 the response at the point $(\theta=0.52 \pi, \phi=0.24 \pi)$ is very small, hence this point should locate within the " $D_{3}$-breaking I" phase. On the other hand, the response at the points $(\theta=0.52 \pi, \phi=$ $0.15 \pi, 0.3 \pi)$ are significant, and they should be within the " $D_{3}$-breaking II" phase.

\section{CONCLUSIONS}

In conclusion, we have studied the classical phase diagram of the one-dimensional spin- $S$ Kitaev-HeisenbergGamma model in the region of an antiferromagnetic Kitaev coupling, based on a combination of classical and spin wave analysis. The revealed "Néel" and " $D_{3}$ breaking I, II" phases are in accordance with the spin-
$1 / 2$ case as discussed in Ref. 46. On the other hand, the " $O_{h} \rightarrow D_{3}$ " phase in the absence of the Heisenberg term is not the same as the " $O_{h} \rightarrow D_{4}$ " phase in the spin- $1 / 2$ case. DMRG numerics provide evidence for the " $O_{h} \rightarrow D_{3}$ " symmetry breaking for higher spins including $S=1$ and $3 / 2$, which are consistent with the classical results. We have also obtained analytic expressions of the lowest-lying spin wave mass perturbatively in the vicinity of the hidden $\mathrm{SU}(2)$ symmetric ferromagnetic point.

Acknowledgments We thank H.-Y. Kee for interesting remarks and helpful discussions. WY and IA acknowledge support from NSERC Discovery Grant 04033-2016. AN acknowledges computational resources and services provided by Compute Canada and Advanced Research Computing at the University of British Columbia. AN is supported by the Canada First Research Excellence Fund.

\section{Appendix A: The Hamiltonians in the six-sublattice rotated frame}

In this section, we spell out the terms in the Hamiltonians in different frames. In general, we write the Hamiltonian $H$ as $H=\sum_{j=1}^{L} H_{j, j+1}$ where $H_{j, j+1}$ is the term on the bond between the sites $j$ and $j+1$. The forms of $H_{j, j+1}$ will be written explicitly.

In the unrotated frame, the form of $H_{j, j+1}$ has a two-site periodicity. We have

$$
\begin{aligned}
& H_{2 n+1,2 n+2}=K S_{2 n+1}^{x} S_{2 n+2}^{x}+\Gamma\left(S_{2 n+1}^{y} S_{2 n+2}^{z}+S_{2 n+1}^{z} S_{2 n+2}^{y}\right)+J \vec{S}_{2 n+1} \cdot \vec{S}_{2 n+2}, \\
& H_{2 n+2,2 n+3}=K S_{2 n+2}^{y} S_{2 n+3}^{y}+\Gamma\left(S_{2 n+2}^{z} S_{2 n+3}^{x}+S_{2 n+2}^{x} S_{2 n+3}^{z}\right)+J \vec{S}_{2 n+2} \cdot \vec{S}_{2 n+3} .
\end{aligned}
$$

In the six-sublattice rotated frame, the form of $H_{j, j+1}^{\prime}$ has a three-site periodicity. We have

$$
\begin{aligned}
& H_{3 n+1,3 n+2}^{\prime}=-K S_{3 n+1}^{x} S_{3 n+2}^{x}-\Gamma\left(S_{3 n+1}^{y} S_{3 n+2}^{y}+S_{3 n+1}^{z} S_{3 n+2}^{z}\right)-J\left(S_{3 n+1}^{x} S_{3 n+2}^{x}+S_{3 n+1}^{y} S_{3 n+2}^{z}+S_{3 n+1}^{z} S_{3 n+2}^{y}\right), \\
& H_{3 n+2,3 n+3}^{\prime}=-K S_{3 n+2}^{z} S_{3 n+3}^{z}-\Gamma\left(S_{3 n+2}^{x} S_{3 n+3}^{x}+S_{3 n+2}^{y} S_{3 n+3}^{y}\right)-J\left(S_{3 n+2}^{z} S_{3 n+3}^{z}+S_{3 n+2}^{x} S_{3 n+3}^{y}+S_{3 n+2}^{y} S_{3 n+3}^{x}\right), \\
& H_{3 n+3,3 n+4}^{\prime}=-K S_{3 n+3}^{y} S_{3 n+4}^{y}-\Gamma\left(S_{3 n+3}^{z} S_{3 n+4}^{z}+S_{3 n+3}^{x} S_{3 n+4}^{x}\right)-J\left(S_{3 n+3}^{y} S_{3 n+4}^{y}+S_{3 n+3}^{z} S_{3 n+4}^{x}+S_{3 n+3}^{x} S_{3 n+4}^{z}\right) .
\end{aligned}
$$


Appendix B: Numerical minimization of the classical free energy
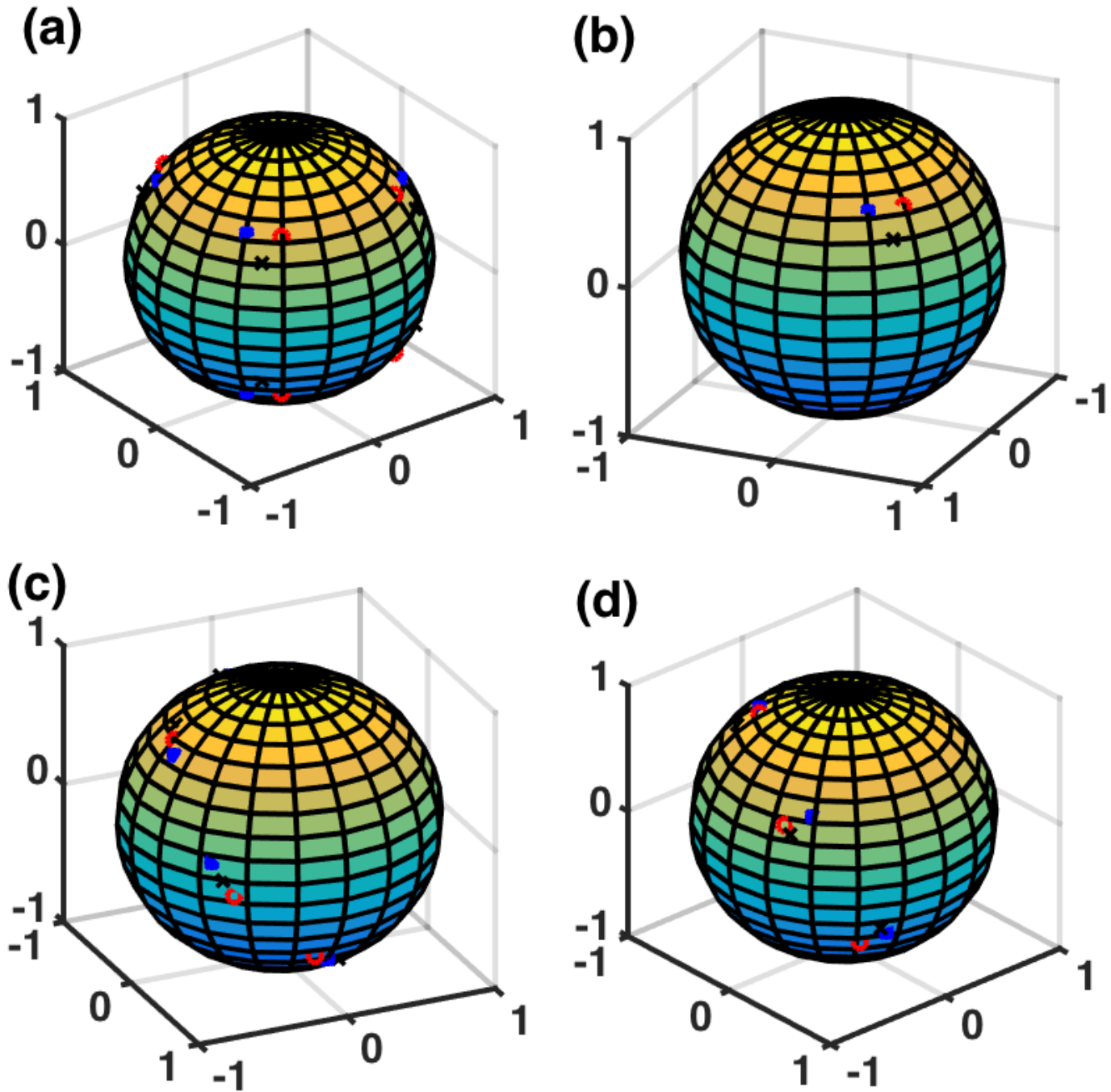

FIG. 13: Classical minima of the free energy at (a) $\varphi=0.15 \pi, J=0 ;$ (b) $\varphi=0.15 \pi, J=0.3 ;(\mathrm{c}) \varphi=0.25 \pi, J=-0.3 ;(\mathrm{d})$ $\varphi=0.15 \pi, J=-0.1$, which lie in the " $O_{h} \rightarrow D_{3}$ " phase, the "Néel" phase, the " $D_{3}$-breaking I" phase and the " $D_{3}$-breaking II" phase, respectively. In (a,b,c,d), the black " $\mathrm{x}$ ", blue " $\times$ " and red "o" symbols represent the spin directions on site $1,2,3$ within unit cell, respectively.

In this appendix, we present the numerical results for minimizing the classical free energies. Throughout this appendix, we work in the six-sublattice rotated frame unless otherwise stated.

In Fig. 13 the classical minima of the free energy are displayed for one representative point in each phase among the " $O_{h} \rightarrow D_{3}$ ", "Neel", " $D_{3}$-breaking I" and " $D_{3}$-breaking II" phases. They all agree with the patterns of the spin alignments discussed in the main text.

We have also compared the classical energies between FM spin configurations (i.e., invariant under $T_{3 a}$ ) and AFM spin configurations (i.e., staggered under $T_{3 a}$ ) for three representative values of $J$, and the results are displayed in Fig. 14. (Note: The FM and AFM here refer to spin alignments in the six-sublattice rotated frame.) As can be seen from Fig. 14, the classical phase transition between FM and AFM occurs at the $\Gamma$ point. The transition point is shifted to larger (smaller) $\phi$ when $J>0(J<0)$. We note that the critical point $\phi_{c}$ is shifted by quantum fluctuations. And what is more, the AFM order for $\phi>\phi_{c}$ may be destroyed by quantum fluctuations. Indeed, as shown in Ref. 44, $\phi_{c}$ at $J=0$ is shifted to $0.33 \pi$ for $S=1 / 2$, and the classical AFM phase does not have any order and the low energy physics is described by the emergent $\mathrm{SU}(2)_{1}$ WZW model. 

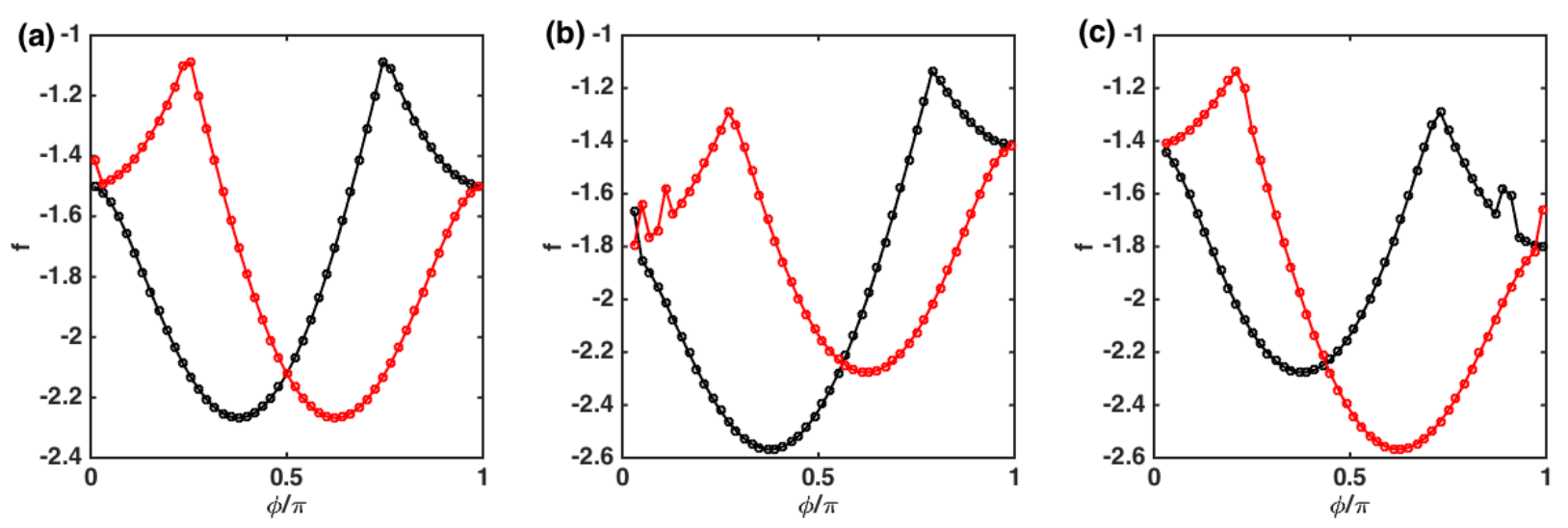

FIG. 14: Classical energies $f$ of $T_{3 a}$-invariant spin configurations (black curve) and $T_{3 a}$-staggered spin configurations (red curve) as functions of $\varphi$ at (a) $J=0$, (b) $J=0.1$, and (c) $J=-0.1$.

\section{Appendix C: Proof of degeneracy}

We give an explanation to the question raised at the end of Sec. IIIA i.e., why the two low-lying eigenvectors are degenerate to all orders in $\Delta$. Although the Hessian matrix $H_{F}(\Delta)$ defined in Eq. (31) always has $O_{h}$ symmetry, the symmetry of the projected Hessian matrix $\mathcal{H}_{F}(\Delta)$ in Eq. (35) is reduced to $D_{3}$ due to the saddle point solutions $r_{i}(\Delta)$ $(i=1,2,3)$ in the definition of the projection matrix $P(\Delta)$. Thus, the eigenspaces of $\mathcal{H}_{F}(\Delta)$ form representations of the group $D_{3}$. Since the $D_{3}$ group only has one- and two-dimensional irreducible representations, generically, we expect one- and two-fold degenerate eigenvalues of $\mathcal{H}_{F}(\Delta)$ except accidental degeneracies. To identify the representations of the eigenspaces (which has to fall into the three irreducible representations of $D_{3}$, i.e., $A_{1}, A_{2}$ and $E$ ), we first consider the $\Delta=0$ case. As can be easily checked, $\left\{v_{1}, v_{2}\right\}$ form the $E$ representation of the $D_{3}$ group, which is two-dimensional. For a nonzero $\Delta$, this irreducible representation cannot be changed unless there is a level crossing. Therefore, we conclude that at least for sufficiently small $\Delta$, the degeneracy of the two low-lying eigenvectors should always be two.

\section{Appendix D: Basics of symplectic linear algebra}

Let $\mathcal{S}$ be a symplectic form on a $2 n$-dimensional linear space. Under a suitable basis, $\mathcal{S}$ acquires the form

$$
\mathcal{S}=\left(\begin{array}{cc}
0 & I_{n} \\
-I_{n} & 0
\end{array}\right)
$$

in which $I_{n}$ is the $n \times n$ identity matrix. A transformation $V$ is called a symplectic transformation if

$$
V \mathcal{S} V^{T}=\mathcal{S}
$$

Let $A$ be a $2 n \times 2 n$ positive-definite real symmetric matrix. Then: (1) the eigenvalues of $\mathcal{S} A$ are all purely imaginary; (2) the eigenvalues appear in pairs as $\pm i \lambda_{j}$ where $\lambda_{j} \in \mathbb{R}$; (3) the eigenvectors satisfy $x^{T} \mathcal{S} y=0$ if $\lambda_{x} \neq-\lambda_{y}$ where $\lambda_{x}, \lambda_{y}$ are the eigenvalues of $x, y$ which are eigenvectors of $\mathcal{S} A$. To see point (1), notice that $A^{1 / 2} \mathcal{S} A^{1 / 2}$ has the same eigenvalues as $\mathcal{S} A$ since they differ by a similar transformation $A^{-1 / 2}(\ldots) A^{1 / 2}$ which is well-defined because $A$ is assumed to be positive-definite. Since $A^{1 / 2} \mathcal{S} A^{1 / 2}$ is antisymmetric, its eigenvalues have to be purely imaginary. For point (2), by taking complex conjugate on both sides of the eigenequation $\mathcal{S} A x=\lambda_{x} x$, it can be seen that $x^{*}$ has eigenvalue $\lambda_{x}^{*}=-\lambda_{x}$. For point (3), notice that on the one hand, $y^{T} A x=y^{T} \mathcal{S}^{T}(\mathcal{S} A x)=-\lambda_{x} y^{T} \mathcal{S} x$; on the other hand, $y^{T} A x=(A y)^{T} x=\left(\mathcal{S}^{T} \mathcal{S} A y\right)^{T} x=\lambda_{y} y^{T} \mathcal{S} x$. This shows that if $\lambda_{x} \neq-\lambda_{y}$, then $y^{T} \mathcal{S} x=0$.

Next we state the central result for our purpose. Let $A$ be positive-definite and real as before. Then there exists a symplectic transformation $V$ (i.e., satisfying Eq. (D2) such that

$$
V \mathcal{S} A V^{T}=\left(\begin{array}{cc}
\Lambda & 0 \\
0 & \Lambda
\end{array}\right)
$$


where $\Lambda$ is a diagonal matrix. We will prove this statement based on the previous discussions. The eigenvectors of $\mathcal{S} A$ are $e_{j} \pm i f_{j}(1 \leq j \leq n)$ with eigenvalues $\pm i \lambda_{j}$, where $e_{j}, f_{j}$ are real vectors. Using $\left(e_{j}^{T}+i \alpha f_{j}^{T}\right) \mathcal{S}\left(e_{k}+i \beta f_{k}\right)=$ $N_{i \alpha} \delta_{j k} \delta_{\alpha,-\beta}(\alpha, \beta= \pm 1)$ where $N_{i \alpha}$ is a normalization factor, it can be verified that

$$
e_{j}^{T} \mathcal{S} e_{k}=f_{j}^{T} \mathcal{S} f_{k}=e_{j}^{T} \mathcal{S} f_{k}=f_{j}^{T} \mathcal{S} e_{k}=0, j \neq k
$$

For $j=k$, the real and imaginary parts of $\left(e_{j}^{T}+i \alpha f_{j}^{T}\right) \mathcal{S}\left(e_{j}+i \alpha f_{j}\right)$ are $e_{j}^{T} \mathcal{S} e_{j}-f_{j}^{T} \mathcal{S} f_{j}$ and $\alpha\left(f_{j}^{T} \mathcal{S} e_{j}+e_{j}^{T} \mathcal{S} f_{j}\right)$, respectively, and both must be vanish according to previous discussions. Furthermore, since $e_{j}^{T} \mathcal{S} e_{j}=\left(e_{j}^{T} \mathcal{S} e_{j}\right)^{T}=$ $-e_{j}^{T} \mathcal{S} e_{j}$, we have $e_{j}^{T} \mathcal{S} e_{j}=f_{j}^{T} \mathcal{S} f_{j}=0$. This shows that the only nonvanishing combinations are $e_{j}^{T} \mathcal{S} f_{j}$ and $f_{j}^{T} \mathcal{S} e_{j}$, which can be normalized to -1 and 1 by a rescaling of $e_{j}, f_{j}$. As a result,

$$
\left(\begin{array}{llllll}
e_{1} & \ldots & e_{n} & f_{1} & \ldots & f_{n}
\end{array}\right)^{T} \mathcal{S}\left(\begin{array}{llllll}
e_{1} & \ldots & e_{n} & f_{1} & \ldots & f_{n}
\end{array}\right)=\left(\begin{array}{cc}
0 & -I_{n} \\
I_{n} & 0
\end{array}\right),
$$

in which the right hand side is just $\mathcal{S}^{T}$. This means that the matrix $U=\left(\begin{array}{llllll}e_{1} & \ldots & e_{n} & f_{1} & \ldots & f_{n}\end{array}\right)$ is a symplectic transformation.

Now we demonstrate that $U$ is able to diagonalize $A$. According to the eigenequations $\mathcal{S} A\left(e_{j} \pm i f_{j}\right)= \pm i \lambda_{j}\left(e_{j} \pm i f_{j}\right)$, we obtain $\mathcal{S} A e_{j}=-\lambda_{j} f_{j}$, and $\mathcal{S} A f_{j}=\lambda_{j} e_{j}$, i.e.,

$$
\mathcal{S} A U=U\left(\begin{array}{cc}
0 & -\Lambda \\
\Lambda & 0
\end{array}\right)
$$

in which $\Lambda=\operatorname{diag}\left(\lambda_{1}, \ldots, \lambda_{n}\right)$. Next multiplying both sides of Eq. (D6) with $U^{T} \mathcal{S}^{T}$, we obtain

$$
U^{T} A U=U^{T} \mathcal{S}^{T} U\left(\begin{array}{cc}
0 & -\Lambda \\
\Lambda & 0
\end{array}\right)
$$

in which $U^{T} \mathcal{S}^{T} U=\mathcal{S}$ according to Eq. D5. But $\mathcal{S}\left(\begin{array}{cc}0 & -\Lambda \\ \Lambda & 0\end{array}\right)=\left(\begin{array}{cc}\Lambda & 0 \\ 0 & \Lambda\end{array}\right)$, thus

$$
U^{T} A U=\left(\begin{array}{cc}
\Lambda & 0 \\
0 & \Lambda
\end{array}\right)
$$

completing the proof.

\section{Appendix E: Proof of Eq. (63)}

We give a proof of Eq. 63 . Define $M^{(n)}$ in terms of the power expansions as

$$
M(\Delta)=M^{(0)}+M^{(1)}+M^{(2)}+\ldots,
$$

where $M^{(n)}$ is proportional to $\Delta^{n}$. Writing

$$
\mathcal{H}_{F}^{M,(1)}=M^{(0)} \mathcal{H}_{F}^{(1)}+M^{(1)} \mathcal{H}_{F}^{(0)},
$$

we obtain

$$
h^{M,(1)}=P_{1}^{(0)} M^{(0)} \mathcal{H}_{F}^{(1)} P_{1}^{(0)}+P_{1}^{(0)} M^{(1)} \mathcal{H}_{F}^{(0)} P_{1}^{(0)} .
$$

Since $P_{1}^{(0)}$ commutes with $M^{(0)}$, the first term in Eq. $\mathrm{E} 3$ is equal to $M^{(0)} P_{1}^{(0)} h^{(1)} P_{1}^{(0)}$. For the second term in Eq. E3), since $P_{1}^{(0)}$ commutes with $\mathcal{H}_{F}^{(0)}$, the second term is equal to the product of $P_{1}^{(0)} M^{(1)} P_{1}^{(0)}$ and $P_{1}^{(0)} \mathcal{H}_{F}^{(0)} P_{1}^{(0)}$. However, $P_{1}^{(0)} M^{(1)} P_{1}^{(0)}$ vanishes. To see this, recall that $M_{j}(\Delta)$ represents the cross product operation with $\hat{n}_{j}^{(0)}(\Delta)$. Denote $T_{j}\left(\hat{n}_{j}^{(0)}\right)$ to be the tangent space of the unit sphere at $\hat{n}_{j}^{(0)}$, and $P_{1 j}^{(0)}$ to be the projection to $T_{j}\left(\hat{n}_{j}^{(0)}\right)$. Then $M_{j}^{(1)}(\Delta)$ corresponds to the cross product with the vector $\delta n_{j}^{(0)}=n_{j}^{(0)}(\Delta)-n_{j}^{(0)}(\Delta=0)$, which lives in $T_{j}\left(\hat{n}_{j}^{(0)}\right)$. Then clearly, the action of $M_{j}^{(1)} P_{1 j}^{(0)}$ on any vector in the tangent space $T_{j}\left(\hat{n}^{(0)}\right)$ is perpendicular to the tangent space, which means that $P_{1 j}^{(0)} M_{j}^{(1)} P_{1 j}^{(0)}=0$. Hence, $P_{1}^{(0)} M^{(1)} P_{1}^{(0)}=0$. 


\section{Appendix F: Equivalence with the Holstein-Primakoff transformation}

We demonstrate that the calculations in Sec. IIIC based on the path integral formalism are equivalent with the Bogoliubov transformation based on the Holstein-Primakoff transformation.

For site $j$, the coordinate frame in the spin space is set up as $\left\{\hat{n}_{j}^{(0)}, \hat{e}_{\theta}^{(0)}(j), \hat{e}_{\phi}^{(0)}(j)\right\}$. Define the spin components $S_{j}^{\prime \alpha}(\alpha=1,2,3)$ as

$$
\begin{aligned}
S_{j}^{\prime 3} & =\hat{n}_{j}^{(0)} \cdot \vec{S}_{j}, \\
S_{j}^{\prime 1} & =\hat{e}_{\theta}^{(0)}(j) \cdot \vec{S}_{j}, \\
S_{j}^{\prime 2} & =\hat{e}_{\phi}^{(0)}(j) \cdot \vec{S}_{j} .
\end{aligned}
$$

Then by introducing the Holstein-Primakoff boson $\left\{b_{j}, b_{j}^{\dagger}\right\}$, the spin operators $S_{j}^{\prime \alpha}$ can be written as

$$
\begin{aligned}
S_{j}^{\prime 3} & =S-b_{j}^{\dagger} b_{j}, \\
S_{j}^{\prime+} & =\sqrt{2 S-b_{j}^{\dagger} b_{j}} \cdot b_{j}, \\
S_{j}^{\prime-} & =b_{j}^{\dagger} \sqrt{2 S-b_{j}^{\dagger} b_{j}},
\end{aligned}
$$

in which $S_{j}^{\prime \pm}=S_{j}^{\prime 1} \pm i S_{j}^{\prime 2}$. Within the spin wave approximation, we have

$$
\begin{aligned}
& S_{j}^{\prime 3}=S-b_{j}^{\dagger} b_{j}, \\
& S_{j}^{\prime 1} \approx \sqrt{\frac{S}{2}} \cdot\left(b_{j}^{\dagger}+b_{j}\right), \\
& S_{j}^{\prime 2} \approx i \sqrt{\frac{S}{2}} \cdot\left(b_{j}^{\dagger}-b_{j}\right) .
\end{aligned}
$$

Neglecting the quartic terms in the boson operators, it can be shown that Eq. F3 leads to

$$
\sum_{\alpha=1,2,3}\left(S_{j}^{\prime \alpha}\right)^{2}=S(S+1),
$$

which is simply the quantum mechanical value of $\vec{S}_{j}^{2}$. On the other hand, if we take the normal ordered product, then Eq. (F3) leads to

$$
\sum_{\alpha=1,2,3}:\left(S_{j}^{\prime \alpha}\right)^{2}:=S^{2},
$$

which coincides with the classical constraints in Eq. (16). Therefore, the procedure of plugging Eq. (F3) into the Hamiltonian in Eq. (1) and keeping only the quadratic terms in boson operators is entirely equivalent to expanding the Lagrangian in the path integral into quadratic terms in the coordinates $\left\{\chi_{\theta}(j), \chi_{\phi}(j)\right\}$, under the following identification

$$
\begin{aligned}
\chi_{\theta}(j) & =\frac{1}{\sqrt{2}}\left(b_{j}^{\dagger}+b_{j}\right), \\
\chi_{\phi}(j) & =i \frac{1}{\sqrt{2}}\left(b_{j}^{\dagger}-b_{j}\right) .
\end{aligned}
$$

This establishes the equivalence between the two methods. In particular, it also fixes the operator ordering in Eq. 72. Because of Eq. $\mathrm{F} 5$, the operators in Eq. 72 should be understood as normal ordered in terms of $\left\{b_{j}, b_{j}^{\dagger}\right\}$.

\section{Appendix G: Perturbative calculation in the " $D_{3}$-breaking I" phase}

In this appendix, we calculate the lowest eigenvalue of the Hessian matrix in the " $D_{3}$ breaking I" phase via third order perturbation theory. We consider the $\Delta=0$ case. In this appendix, we take $\Gamma^{\prime}=1$ for simplification of notation. 


\section{First order perturbation}

Let

$$
x=-\frac{1}{\sqrt{2}}+x^{\prime}, y=y^{\prime}, z=\frac{1}{2}+z^{\prime}, \lambda_{1}=-2+\lambda_{1}^{\prime}, \lambda_{2}=-2+\lambda_{2}^{\prime} .
$$

Then Eq. 87 becomes

$$
\begin{aligned}
2 x^{\prime}+z^{\prime}+\frac{1}{\sqrt{2}} \lambda_{1}^{\prime}-|J| x^{\prime}-\lambda_{1}^{\prime} x^{\prime} & =0 \\
3 y^{\prime}+\frac{1}{\sqrt{2}}|J|-|J| y^{\prime}-\lambda_{1}^{\prime} y^{\prime} & =0 \\
x^{\prime}+2 z^{\prime}-\frac{1}{\sqrt{2}}|J|-\frac{1}{\sqrt{2}} \lambda_{1}^{\prime}-|J| z^{\prime}-\lambda_{1}^{\prime} z^{\prime} & =0 \\
-x^{\prime}+z^{\prime}+\frac{1}{\sqrt{2}} \lambda_{2}^{\prime}-\frac{1}{\sqrt{2}}|J|+|J| x^{\prime}-|J| y^{\prime} & =0 \\
-\sqrt{2} x^{\prime}+\sqrt{2} z^{\prime}+x^{\prime 2}+y^{\prime 2}+z^{\prime 2} & =0 .
\end{aligned}
$$

The quantities $x^{\prime}, y^{\prime}, z^{\prime}, \lambda_{1}^{\prime}, \lambda_{2}^{\prime}$ can be expanded in a power expansion in $J$, i.e.,

$$
x^{\prime}=\sum_{n \geq 1} x^{(n)}, y^{\prime}=\sum_{n \geq 1} y^{(n)}, z^{\prime}=\sum_{n \geq 1} z^{(n)}, \lambda_{1}^{\prime}=\sum_{n \geq 1} \lambda_{1}^{(n)}, \lambda_{2}^{\prime}=\sum_{n \geq 1} \lambda_{2}^{(n)},
$$

in which $x^{(n)}, y^{(n)}, z^{(n)}, \lambda_{1}^{(n)}, \lambda_{2}^{(n)}$ are all proportional to $J^{n}$.

Plugging Eq. G3 into Eq. 87] and keeping terms only up to $O(J)$, we obtain

$$
\begin{array}{r}
2 x^{(1)}+z^{(1)}+\frac{1}{\sqrt{2}} \lambda_{1}^{(1)}=0 \\
3 y^{(1)}+\frac{1}{\sqrt{2}}|J|=0 \\
x^{(1)}+2 z^{(1)}-\frac{1}{\sqrt{2}} \lambda_{1}^{(1)}-\frac{1}{\sqrt{2}}|J|=0 \\
-x^{(1)}+z^{(1)}+\frac{1}{\sqrt{2}} \lambda_{2}^{(1)}-\frac{1}{\sqrt{2}}|J|=0 \\
-\sqrt{2} x^{(1)}+\sqrt{2} z^{(1)}=0 .
\end{array}
$$

The solution of Eq. G4 gives

$$
\begin{aligned}
& \vec{r}_{1}=\left(-\frac{1}{\sqrt{2}}+\frac{1}{6 \sqrt{2}}|J|,-\frac{1}{3 \sqrt{2}}|J|, \frac{1}{\sqrt{2}}+\frac{1}{6 \sqrt{2}}|J|\right)^{T}, \\
& \vec{r}_{2}=\left(-\frac{1}{\sqrt{2}}, 0, \frac{1}{\sqrt{2}}\right)^{T}, \\
& \vec{r}_{3}=\left(-\frac{1}{\sqrt{2}}-\frac{1}{6 \sqrt{2}}|J|, \frac{1}{3 \sqrt{2}}|J|, \frac{1}{\sqrt{2}}-\frac{1}{6 \sqrt{2}}|J|\right)^{T}, \\
& \lambda_{1}=-2-\frac{1}{2}|J|, \\
& \lambda_{2}=-2+|J|,
\end{aligned}
$$

in which $\vec{S}_{i}=S \vec{r}_{i}, i=1,2,3$.

To lowest order in $J$, the above equations reduce to Eq. G4.

Notice that as discussed in Sec. VB2, the smallest spin wave mass vanishes in first order perturbation. 


\section{Second order perturbation}

Now we expand up to $O\left(J^{2}\right)$, then Eq. G2 becomes

$$
\begin{aligned}
2 x^{(2)}+z^{(2)}+\frac{1}{\sqrt{2}} \lambda_{1}^{(2)} & =|J| x^{(1)}+\lambda_{1}^{(1)} x^{(1)}=\frac{1}{12 \sqrt{2}} J^{2} \\
3 y^{(2)} & =|J| y^{(1)}+\lambda_{1}^{(1)} y^{(1)}=-\frac{1}{6 \sqrt{2}} J^{2} \\
x^{(2)}+2 z^{(2)}-\frac{1}{\sqrt{2}} \lambda_{1}^{(2)} & =\left(|J|+\lambda_{1}^{(1)}\right) z^{(1)}=\frac{1}{12 \sqrt{2}} J^{2} \\
-x^{(2)}+z^{(2)}+\frac{1}{\sqrt{2}} \lambda_{2}^{(2)} & =|J|\left(-x^{(1)}+y^{(1)}\right)=-\frac{1}{2 \sqrt{2}} J^{2} \\
\sqrt{2} x^{(2)}-\sqrt{2} z^{(2)} & =\left(x^{(1)}\right)^{2}+\left(y^{(1)}\right)^{2}+\left(z^{(1)}\right)^{2}=\frac{1}{12} J^{2} .
\end{aligned}
$$

The solutions are

$$
x^{(2)}=\frac{5}{72 \sqrt{2}} J^{2}, y^{(2)}=-\frac{1}{18 \sqrt{2}} J^{2}, z^{(2)}=-\frac{1}{72 \sqrt{2}} J^{2}, \lambda_{1}^{(2)}=-\frac{1}{24 \sqrt{2}} J^{2}, \lambda_{2}^{(2)}=-\frac{5}{12 \sqrt{2}} J^{2} .
$$

From this, we are able to expand $\Delta \mathcal{H}_{F}(J)$ as $\Delta \mathcal{H}_{F}(J)=\Delta \mathcal{H}_{F}^{(1)}(J)+\Delta \mathcal{H}_{F}^{(2)}(J)$. Let $\Delta \mathcal{H}_{\text {red }}^{(2)}(J)$ be the projection of the following matrix

$$
\Delta \mathcal{H}_{F}^{(2)}(J)+\Delta \mathcal{H}_{F}^{(1)}(J) \sum_{i=1}^{4} \frac{w_{i}^{T} w_{i}}{E_{0}-E_{i}} \Delta \mathcal{H}_{F}^{(1)}(J)
$$

to the subspace spanned by $v_{1}, v_{2}$, in which $w_{i}(i=1,2,3,4)$ are given in Eq. (33) where

$$
\hat{e}_{\theta}=\left(-\frac{1}{\sqrt{2}}, 0,-\frac{1}{\sqrt{2}}\right)^{T}, \hat{e}_{\phi}=(0,-1,0)^{T}
$$

and $E_{i}=3$ are the eigenvalues of $w_{i}$. Calculations show that $\Delta \mathcal{H}_{\text {red }}^{(2)}(J)=0$, which means that we have to go to third order.

Notice that as discussed in Sec. VB2, the smallest spin wave mass still vanishes in second order perturbation.

\section{Third order perturbation}

The third order expansion of Eq. G2 gives

$$
\begin{aligned}
2 x^{(3)}+z^{(3)}+\frac{1}{\sqrt{2}} \lambda_{1}^{(3)} & =|J| x^{(2)}+\lambda_{1}^{(1)} x^{(2)}+\lambda_{2}^{(2)} x^{(1)}=\frac{1}{36 \sqrt{2}}|J|^{3} \\
3 y^{(3)} & =\left(|J|+\lambda_{1}^{(1)}\right) y^{(2)}+\lambda_{1}^{(2)} y^{(1)}=-\frac{1}{72 \sqrt{2}}|J|^{3} \\
x^{(3)}+2 z^{(3)}-\frac{1}{\sqrt{2}} \lambda^{(1)} & =|J| z^{(2)}+\lambda_{1}^{(1)} z^{(2)}+\lambda_{1}^{(2)} z^{(1)}=-\frac{1}{72 \sqrt{2}}|J|^{3} \\
\lambda_{2}^{(3)}-\sqrt{2} x^{(3)}+\sqrt{2} z^{(3)} & =-\sqrt{2}|J| x^{(2)}+\sqrt{2}|J| y^{(2)}=\frac{1}{8}|J|^{3} \\
x^{(3)}-z^{(3)} & =\sqrt{2}\left(x^{(1)} x^{(2)}+y^{(1)} y^{(2)}+z^{(1)} z^{(2)}\right)=\frac{1}{36 \sqrt{2}}|J|^{3} .
\end{aligned}
$$

The solution is

$$
x^{(3)}=\frac{7}{432 \sqrt{2}}|J|^{3}, y^{(3)}=-\frac{1}{216 \sqrt{2}}|J|^{3}, z^{(3)}=-\frac{5}{432 \sqrt{2}}|J|^{3}, \lambda_{1}^{(3)}=\frac{1}{144}|J|^{3}, \lambda_{2}^{(3)}=-\frac{7}{72}|J|^{3} .
$$

From this, we are able to obtain $\Delta \mathcal{H}_{F}^{(3)}(J)$ from the expansion $\Delta \mathcal{H}_{F}(J)=\Delta \mathcal{H}_{F}^{(1)}(J)+\Delta \mathcal{H}_{F}^{(2)}(J)+\Delta \mathcal{H}_{F}^{(3)}(J)$. 
The third order perturbation matrix is given by

$$
\begin{aligned}
& h^{(3)}(J)=P_{1}^{(0)}\left[\Delta \mathcal{H}_{F}^{(3)}(J)+\Delta \mathcal{H}_{F}^{(1)}(J) \sum_{i=1}^{4} \frac{w_{i}^{T} w_{i}}{E_{0}-E_{i}} \Delta \mathcal{H}_{F}^{(2)}(J)+\Delta \mathcal{H}_{F}^{(2)}(J) \sum_{i=1}^{4} \frac{w_{i}^{T} w_{i}}{E_{0}-E_{i}} \mathcal{H}_{F}^{(1)}(J)\right. \\
& \left.+\sum_{1 \leq i, j \leq 4} \frac{\Delta \mathcal{H}_{F}^{(1)}(J) w_{i} w_{i}^{T} \Delta \mathcal{H}_{F}^{(1)}(J) w_{j} w_{j}^{T} \Delta \mathcal{H}_{F}^{(1)}(J)}{\left(E_{0}-E_{i}\right)\left(E_{0}-E_{j}\right)}-\sum_{1 \leq i \leq 2,1 \leq j \leq 4} \frac{\Delta \mathcal{H}_{F}^{(1)}(J) v_{i} v_{i}^{T} \Delta \mathcal{H}_{F}^{(1)}(J) w_{j} w_{j}^{T} \Delta \mathcal{H}_{F}^{(1)}(J)}{\left(E_{0}-E_{j}\right)^{2}}\right] P_{1}^{(0)}
\end{aligned}
$$

Recall that the degeneracy has already been broken within first order perturbation theory. The vector up to zeroth order is

$$
\psi=-\sqrt{\frac{2}{3}} v_{1}+\frac{1}{\sqrt{3}} v_{2} .
$$

Then the energy correction at $O\left(J^{3}\right)$ can be directly obtained by $\psi^{T} h^{(3)}(J) \psi$, which is $\frac{1}{2}|J|^{3}$.

Next, we proceed to calculate the spin wave mass. The perturbation matrix at $O\left(J^{3}\right)$ is given by

$$
\begin{aligned}
& h^{M,(3)}(J)=P_{1}^{(0)}\left[\Delta \mathcal{H}_{F}^{M,(3)}(J)+\Delta \mathcal{H}_{F}^{M,(1)}(J) \sum_{i=1}^{4} \frac{u_{i}^{\dagger} u_{i}}{E_{0}-\epsilon_{i}} \Delta \mathcal{H}_{F}^{M,(2)}(J)+\Delta \mathcal{H}_{F}^{M,(2)}(J) \sum_{i=1}^{4} \frac{u_{i}^{\dagger} u_{i}}{E_{0}-\epsilon_{i}} \mathcal{H}_{F}^{M,(1)}(J)\right. \\
& \left.+\sum_{1 \leq i, j \leq 4} \frac{\Delta \mathcal{H}_{F}^{M,(1)}(J) u_{i} u_{i}^{\dagger} \Delta \mathcal{H}_{F}^{M,(1)}(J) u_{j} u_{j}^{\dagger} \Delta \mathcal{H}_{F}^{M,(1)}(J)}{\left(E_{0}-\epsilon_{i}\right)\left(E_{0}-\epsilon_{j}\right)}-\sum_{1 \leq i \leq 2,1 \leq j \leq 4} \frac{\Delta \mathcal{H}_{F}^{M,(1)}(J) v_{i} v_{i}^{\dagger} \Delta \mathcal{H}_{F}^{M,(1)}(J) u_{j} u_{j}^{\dagger} \Delta \mathcal{H}_{F}^{(1)}(J)}{\left(E_{0}-\epsilon_{j}\right)^{2}}\right] P_{1}^{(0)}
\end{aligned}
$$

Evaluation of Eq. G14 gives Eq. 100. To obtain $m_{1}$, we need to calculate the eigenvalues of $h^{M,(1)}(J)+h^{M,(3)}(J)$ (recall that $\left.h^{M,(2)}(J)=0\right)$. Calculations show that the eigenvalues are $\pm i J^{2}$.

We make a comment here. When $J=0(\Delta=0$ as before $)$, the null space of $M(\Delta=0, J=0) \mathcal{H}_{F}(\Delta=0, J=0)$ is five-dimensional, and the corresponding eigenvectors are $v_{i}(i=1,2)$ and $r_{j}(j=1,2,3)$, where $r_{j}$ 's are given by Eq. 30 in which $\hat{n}_{j}^{(0)}=\left(-\frac{1}{\sqrt{2}}, 0, \frac{1}{\sqrt{2}}\right)$. Rigorously, we should perform a degenerate perturbation theory in this five-dimensional space, instead of a perturbation within the two-dimensional space spanned by $\left\{v_{1}, v_{2}\right\}$ as discussed previously. However, we demonstrate that in obtaining the two nonzero eigenvalues $\pm i J^{2}$, it is enough to work within the two-dimensional space. The perturbation matrix $h_{5}^{M,(1,2,3)}(J)$ in the five-dimensional space spanned by $\left\{v_{1}, v_{2}, r_{1}, r_{2}, r_{3}\right\}$ up to third order can be obtained by replacing $P_{1}^{(0)}$ in Eqs. 9597 G12 with the projection to the five-dimensional space. The result is

$$
h_{5}^{M,(1,2,3)}(J)=\left(\begin{array}{ccccc}
-\frac{2 \sqrt{2}}{3}|J|+\frac{19}{54 \sqrt{2}}|J|^{3} & -\frac{2}{3}|J|-\frac{35}{108}|J|^{3} & 0 & 0 & 0 \\
\frac{4}{3}|J|+\frac{4}{27}|J|^{3} & \frac{2 \sqrt{2}}{3}|J|-\frac{19}{54 \sqrt{2}}|J|^{3} & 0 & 0 & 0 \\
-\frac{1}{3} \sqrt{\frac{2}{3}}|J|^{2}-\frac{1}{9 \sqrt{6}}|J|^{3} & -\frac{1}{3 \sqrt{3}}|J|^{2}-\frac{1}{18 \sqrt{3}}|J|^{3} & 0 & 0 & 0 \\
0 & 0 & 0 & 0 & 0 \\
\frac{1}{3} \sqrt{\frac{2}{3}}|J|^{2}+\frac{1}{9 \sqrt{6}}|J|^{3} & \frac{1}{3 \sqrt{3}}|J|^{2}+\frac{1}{18 \sqrt{3}}|J|^{3} & 0 & 0 & 0
\end{array}\right) .
$$

As can seen from Eq. (G15), to get the two nonzero eigenvalues, it is enough to consider an eigenvalue problem of the upper-left $2 \times 2$ block, since the vectors $r_{j}(j=1,2,3)$ always lie within the null space of the matrix in Eq. G15 regardless of the value of $J$. The eigenvalues $\pm i J^{2}$ are obtained in this way, i.e., by calculating the eigenvalues of the upper-left $2 \times 2$ block. We also note that the eigenvectors of the two nonzero eigenvalues $\pm i J^{2}$ contain components on $r_{j}(j=1,2,3)$ due to the nonzero matrix elements in the third, fourth, and fifth row (but only within the first and second columns) of $h_{5}^{M,(1,2,3)}(J)$.

\section{Appendix H: Perturbative calculation in the " $D_{3}$-breaking II" phase}

Here the zeroth order solution can be taken as the one along the $(1,-1,1)$-direction:

$$
\begin{aligned}
& \hat{n}_{1}^{(0)}=\frac{1}{\sqrt{3}}\left(x_{0},-x_{0}, z_{0}\right)^{T}, \hat{n}_{2}^{(0)}=\frac{1}{\sqrt{3}}\left(x_{0},-z_{0}, x_{0}\right)^{T}, \hat{n}_{3}^{(0)}=\frac{1}{\sqrt{3}}\left(z_{0},-x_{0}, x_{0}\right)^{T}, \\
& \lambda_{1}^{(0)}=\lambda_{2}^{(0)}=\lambda_{3}^{(0)}=\lambda_{0},
\end{aligned}
$$



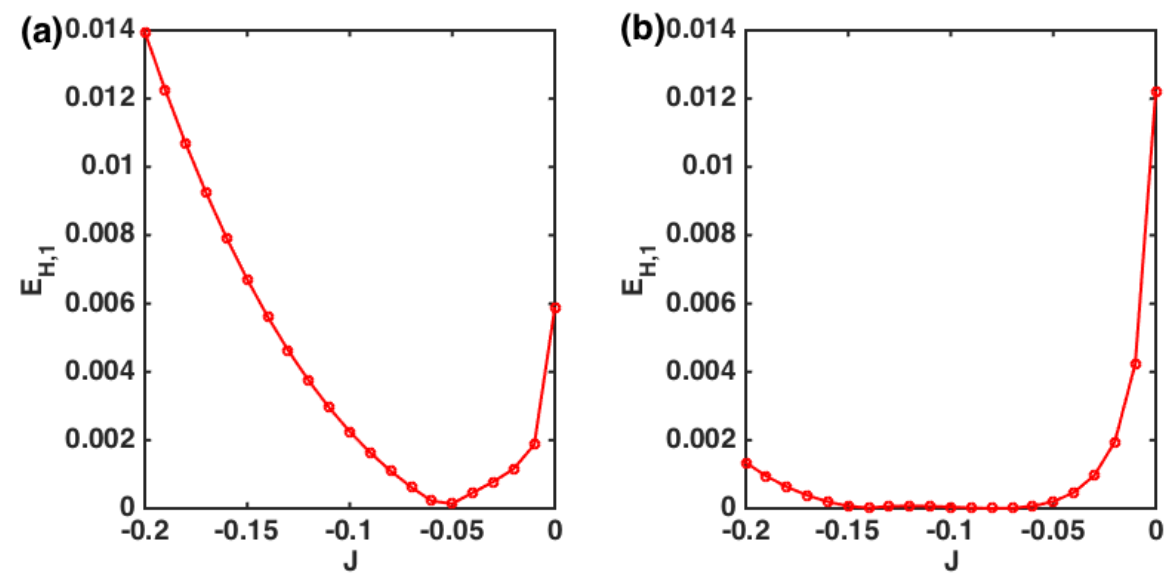

FIG. 15: Smallest eigenvalue vs. $J$ for (a) $\varphi=0.21 \pi$, and (b) $\varphi=0.30 \pi$, where in accordance with the main text, $\varphi$ is defined through the parametrization $K=\cos (\varphi), \Gamma=\sin (\varphi)$. Notice that although when $\varphi<0.25 \pi$, the dependence of the smallest eigenvalue on $J$ is regular, the eigenvalue exhibits a rather complicated behavior when $\varphi>0.25 \pi$. On the other hand, as discussed in the main text, the value of $m_{1}$ is regular even for $\varphi>0.25 \pi$.

in which

$$
x_{0}=1+\frac{1}{9} \Delta-\frac{2}{81} \Delta^{2}, z_{0}=1-\frac{2}{9} \Delta+\frac{1}{81} \Delta^{2}, \lambda_{0}=-2-\frac{2}{3} \Delta-\frac{2}{27} \Delta^{2} .
$$

We solve the saddle point equations perturbatively in an expansion over $J$ starting with a nonzero $\Delta$. There is some difficulty in calculating the eigenvalues of the Hessian matrix. Instead of deriving a perturbative result, we study the eigenvalues numerically. In this appendix, we take $\Gamma^{\prime}=1$ for simplification of notation.

Let

$$
\begin{gathered}
x=\frac{1}{\sqrt{3}}\left(x_{0}+x^{\prime}\right), y=\frac{1}{\sqrt{3}}\left(-x_{0}+y^{\prime}\right), z=\frac{1}{\sqrt{3}}\left(z_{0}+z^{\prime}\right), m=\frac{1}{\sqrt{3}}\left(x_{0}+m^{\prime}\right), n=\frac{1}{\sqrt{3}}\left(-z_{0}+n^{\prime}\right), \\
\lambda_{1}=\lambda_{0}+\lambda_{1}^{\prime}, \lambda_{2}=\lambda_{0}+\lambda_{2}^{\prime} .
\end{gathered}
$$

in which the primed variables are assumed to be $O(J)$. Plugging these into the saddle point equations and only keeping the $O(J)$ terms, we obtain

$$
\left(\begin{array}{ccccccc}
-\lambda_{0} & 0 & -1 & -(1+\Delta) & 0 & -x_{0} & 0 \\
0 & -\left(1+\Delta+\lambda_{0}\right) & 0 & 0 & -1 & x_{0} & 0 \\
-1 & 0 & -\lambda_{0} & -1 & 0 & -z_{0} & 0 \\
-(1+\Delta) & 0 & -1 & -\lambda_{0} & 0 & 0 & -x_{0} \\
0 & -2 & 0 & 0 & -\lambda_{0} & 0 & z_{0} \\
x_{0} & -x_{0} & z_{0} & 0 & 0 & 0 & 0 \\
0 & 0 & 0 & 2 x_{0} & -z_{0} & 0 & 0
\end{array}\right)\left(\begin{array}{c}
x^{\prime} \\
y^{\prime} \\
z^{\prime} \\
m^{\prime} \\
n^{\prime} \\
\lambda_{1}^{\prime} \\
\lambda_{2}^{\prime}
\end{array}\right)=\left(\begin{array}{c}
-2 x_{0}|J| \\
0 \\
0 \\
0 \\
-2 z_{0}|J| \\
0 \\
0
\end{array}\right)
$$

The solution gives Eqs. 103 104 105.

Next we try to proceed as before by defining $\mathcal{H}_{F}(\Delta, J)=P(\Delta, J) H_{F}(\Delta, J) P(\Delta, J)$, and $\Delta \mathcal{H}_{F}(\Delta, J)=\mathcal{H}_{F}(\Delta, J)-$ $\mathcal{H}_{F}(\Delta, J=0)$. Consider the first order degenerate perturbation

$$
h^{(1)}(\Delta, J)=\left(\begin{array}{cc}
v_{1}^{T} \Delta \mathcal{H}_{F}(\Delta, J) v_{1} & v_{1}^{T} \Delta \mathcal{H}_{F}(\Delta, J) v_{2} \\
v_{2}^{T} \Delta \mathcal{H}_{F}(\Delta, J) v_{1} & v_{2}^{T} \Delta \mathcal{H}_{F}(\Delta, J) v_{2}
\end{array}\right) .
$$

This time, the leading order contribution is $O\left(\bar{J} / \Delta^{2}\right)$. On the other hand, calculations show that the leading nonvanishing terms in $h^{(1)}(\Delta, J)$ is $O(J)$. However, if we want to reach $O(J)$, the calculations in Eq. H5 are not enough. Let $v_{1}(\Delta)$ and $v_{2}(\Delta)$ be the two lowest spin wave vectors at $J=0$. Since $\Delta \mathcal{H}_{F}(\Delta, J)$ contains $O\left(1 / \Delta^{2}\right)$ terms, we have to keep $v_{i}(\Delta)(i=1,2)$ to $O\left(\Delta^{2}\right)$ so that $O(J)$ can be reached for $h^{(1)}(\Delta, J)$. Recall that up to $O\left(\Delta^{2}\right)$, the eigenvalues are still degenerate for $v_{i}(\Delta)(i=1,2)$, both equal to $\frac{4}{27} \Delta^{2}$. The best situation is that they split in the third order perturbation, i.e., to $O\left(\Delta^{3}\right)$. Then we have linear combinations $\alpha_{1} v_{1}+\beta_{1} v_{2}$ and $\alpha_{2} v_{1}+\beta_{2} v_{2}$, with an energy difference $\sim O\left(\Delta^{3}\right)$. To get an $O\left(\Delta^{2}\right)$ mixture between $\alpha_{i} v_{1}+\beta_{i} v_{2}(i=1,2)$, we have to go to another two orders of perturbations, i.e., fifth order perturbation in $\Delta$.

The smallest eigenvalue is calculated by numerics shown in Fig. 15. As can be seen from Fig. 15, the results show a very complicated behavior when $\phi>0.25 \pi$. 
Appendix I: Numerical results in the " $D_{3}$-breaking I, II" phases for $S=3 / 2$

Fig. 16 shows the results for the spin expectation vales $\left\langle S_{j}^{\alpha}\right\rangle(\alpha=x, y, z)$ at three representative points $(\theta=$ $0.52 \pi, \phi=0.15 \pi),(\theta=0.52 \pi, \phi=0.25 \pi)$ and $(\theta=0.52 \pi, \phi=0.30 \pi)$ under the $h_{\mathrm{I}}$ and $h_{\mathrm{II}}$ fields for the $S=3 / 2$ case. ED numerics are performed on a system of $L=18$ sites with periodic boundary conditions, and both $h_{\mathrm{I}}$ and $h_{\mathrm{II}}$ fields are taken to be $10^{-4}$. As can be clearly seen from Figs. 10 , the spin alignments are consistent with the patterns given in Eqs. 8586, thereby confirming the existence of the " $D_{3}$-breaking I, II" phases for $S=3 / 2$.
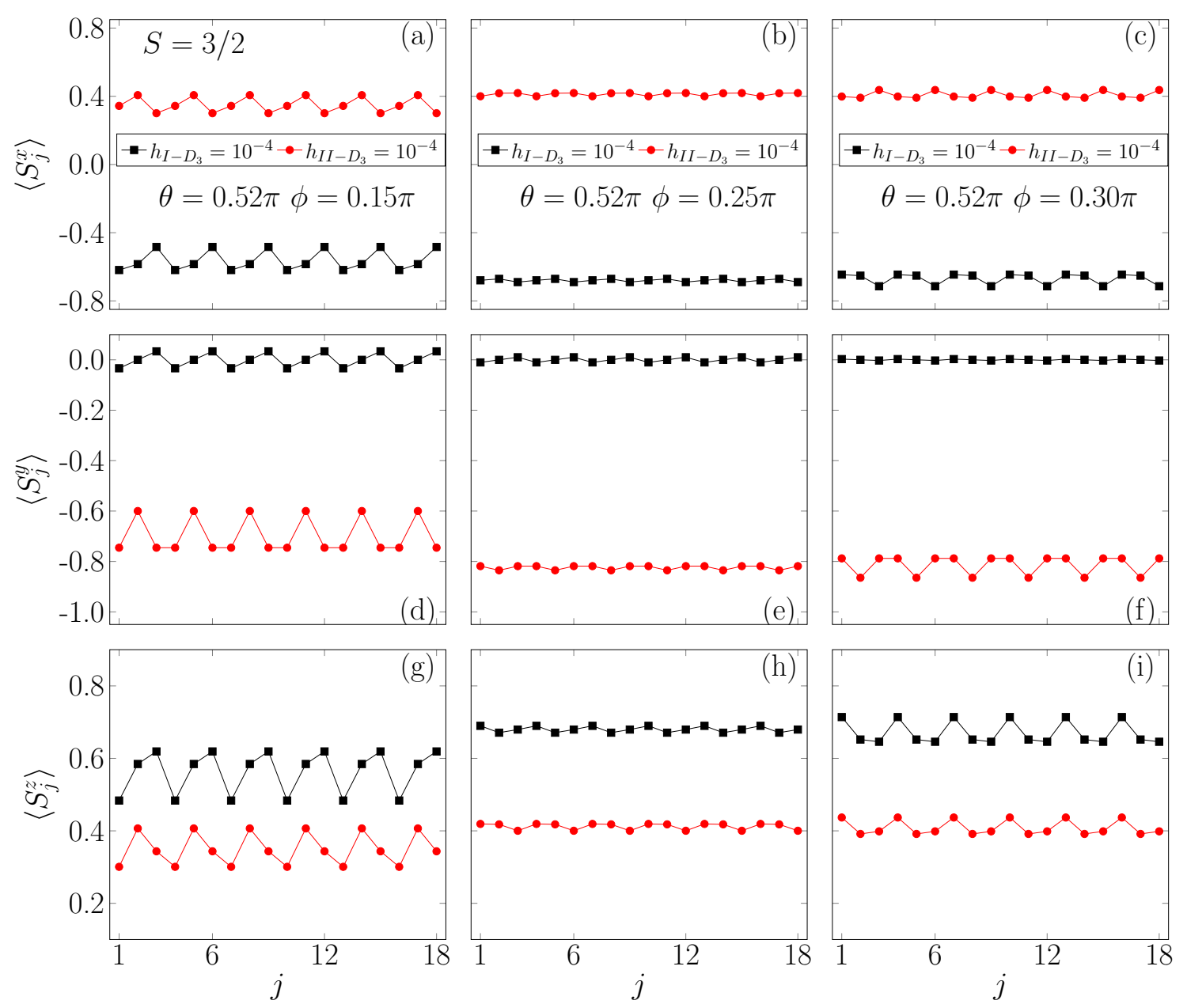

FIG. 16: (a,b,c) $\left\langle S_{j}^{x}\right\rangle$, (d,e,f) $\left\langle S_{j}^{y}\right\rangle$, and (g,h,i) $\left\langle S_{j}^{z}\right\rangle$ vs $j$ under $h_{\mathrm{I}}$ (black squares) and $h_{\mathrm{II}}$ (red dots) fields for $S=3 / 2$ at several different points. (a,d,g) are for $(\theta=0.52 \pi, \phi=0.15 \pi)$; (b,e,h) for $(\theta=0.52 \pi, \phi=0.25 \pi)$; and $(\mathrm{c}, \mathrm{f}, \mathrm{i})$ for $(\theta=0.52 \pi, \phi=0.30 \pi)$. DMRG numerics are performed on $L=18$ sites with periodic boundary conditions. Both $h_{\mathrm{I}}$ and $h_{\mathrm{II}}$ fields are taken to be $10^{-4}$.

1 P. Fazekas, Lecture Notes on Electron Correlation and Magnetism (World Scientific, Singapore, 1999).

2 A. Laüchli, F. Mila, and K. Penc, Phys. Rev. Lett. 97, 087205 (2006).

${ }^{3}$ L. Balents, Nature 464, 199 (2010).

${ }^{4}$ W. Witczak-Krempa, G. Chen, Y. B. Kim, and L. Balents, Annu. Rev. Condens. Matter Phys. 5, 57 (2014).

5 J. G. Rau, E. K.-H. Lee, and H.-Y. Kee, Annu. Rev. Con- dens. Matter Phys. 7, 195 (2016).

6 L. Savary and L. Balents, Reports Prog. Phys. 80, 016502 (2017).

7 S. M. Winter, A. A. Tsirlin, M. Daghofer, J. van den Brink, Y. Singh, P. Gegenwart, and R. Valentí, J. Phys. Condens. Matter 29, 493002 (2017).

8 Y. Zhou, K. Kanoda, and T.-K. Ng, Rev. Mod. Phys. 89, 025003 (2017). 
9 G. Jackeli and G. Khaliullin, Phys. Rev. Lett. 102, 017205 (2009).

10 J. Chaloupka, G. Jackeli, and G. Khaliullin, Phys. Rev. Lett. 105, 027204 (2010).

11 J. G. Rau, E. K.-H. Lee, and H.-Y. Kee, Phys. Rev. Lett. 112, 077204 (2014).

12 A. Kitaev, Ann. Phys. (N. Y). 321, 2 (2006).

13 Y. Singh and P. Gegenwart, Phys. Rev. B 82, 064412 (2010).

14 J. Reuther, R. Thomale, and S. Trebst, Phys. Rev. B 84, 23100406 (2011).

15 H.-C. Jiang, Z.-C. Gu, X.-L. Qi, and S. Trebst, Phys. Rev. B 83, 245104 (2011).

16 C. C. Price and N. B. Perkins, Phys. Rev. Lett. 109, 187201 (2012).

17 S. K. Choi, R. Coldea, A. N. Kolmogorov, T. Lancaster, I. I. Mazin, S. J. Blundell, P. G. Radaelli, Y. Singh, P. Gegenwart, K. R. Choi, et al., Phys. Rev. Lett. 108, 127204 (2012).

18 Y. Singh, S. Manni, J. Reuther, T. Berlijn, R. Thomale, W. Ku, S. Trebst, and P. Gegenwart, Phys. Rev. Lett. 108, 127203 (2012).

19 J. Chaloupka, G. Jackeli, and G. Khaliullin, Phys. Rev. Lett. 110, 097204 (2013).

${ }^{20}$ K. A. Modic, T. E. Smidt, I. Kimchi, N. P. Breznay, A. Biffin, S. Choi, R. D. Johnson, R. Coldea, P. Watkins-Curry, G. T. McCandless, J. Y. Chan, F. Gandara, Z. Islam, A. Vishwanath, A. Shekhter, R. D. McDonald, and J. G. Analytis, Nat. Commun. 5, 4203 (2014).

${ }^{21}$ K. W. Plumb, J. P. Clancy, L. J. Sandilands, V. V. Shankar, Y. F. Hu, K. S. Burch, H.-Y. Kee, and Y.-J. Kim, Phys. Rev. B 90, 041112 (2014).

22 H.-S. Kim, V. S. V., A. Catuneanu, and H.-Y. Kee, Phys. Rev. B 91, 241110 (2015).

${ }^{23}$ R. D. Johnson, S. C. Williams, A. A. Haghighirad, J. Singleton, V. Zapf, P. Manuel, I. I. Mazin, Y. Li, H. O. Jeschke, R. Valentí, and R. Coldea, Phys. Rev. B 92, 235119 (2015).

${ }^{24}$ L. J. Sandilands, Y. Tian, K. W. Plumb, Y.-J. Kim, and K. S. Burch, Phys. Rev. Lett. 114, 147201 (2015).

25 J. A. Sears, M. Songvilay, K. W. Plumb, J. P. Clancy, Y. Qiu, Y. Zhao, D. Parshall, and Y.-J. Kim, Phys. Rev. B 91, 144420 (2015).

26 A. Banerjee, C. A. Bridges, J.-Q. Yan, A. A. Aczel, L. Li, M. B. Stone, G. E. Granroth, M. D. Lumsden, Y. Yiu, J. Knolle, S. Bhattacharjee, D. L. Kovrizhin, R. Moessner, D. A. Tennant, D. G. Mandrus, and S. E. Nagler, Nat. Mater. 15, 733 (2016)

27 R. Yadav, N. A. Bogdanov, V. M. Katukuri, S. Nishimoto, J. van den Brink, and L. Hozoi, Sci. Rep. 6, 37925 (2016).

28 S.-H. Baek, S.-H. Do, K.-Y. Choi, Y. Kwon, A. Wolter, S. Nishimoto, J. van den Brink, and B. Büchner, Phys. Rev. Lett. 119, 037201 (2017).

29 A. Banerjee, J. Yan, J. Knolle, C. A. Bridges, M. B. Stone, M. D. Lumsden, D. G. Mandrus, D. A. Tennant, R. Moessner, and S. E. Nagler, Science 356, 1055 (2017).

30 J. Zheng, K. Ran, T. Li, J. Wang, P. Wang, B. Liu, Z.-X. Liu, B. Normand, J. Wen, and W. Yu, Phys. Rev. Lett. 119, 227208 (2017)

${ }^{31}$ K. Ran, J. Wang, W. Wang, Z.-Y. Dong, X. Ren, S. Bao, S. Li, Z. Ma, Y. Gan, Y. Zhang, J.?T. Park, G. Deng, S. Danilkin, S.-L. Yu, J.-X. Li, and J. Wen, Phys. Rev. Lett. 118, 107203 (2017)

32 C. Wang, A. Nahum, M. A. Metlitski, C. Xu, and T.
Senthil, Phys. Rev. X 7, 031051 (2017).

${ }^{33}$ L. Janssen, E. C. Andrade, and M. Vojta, Phys. Rev. B 96, 064430 (2017).

34 Z.-X. Liu and B. Normand, Phys. Rev. Lett. 120, 187201 (2018).

35 A. Catuneanu, Y. Yamaji, G. Wachtel, Y. B. Kim, and H.-Y. Kee, npj Quantum Mater. 3, 23 (2018).

36 M. Gohlke, G. Wachtel, Y. Yamaji, F. Pollmann, and Y. B. Kim, Phys. Rev. B 97, 075126 (2018).

37 N. Janša, A. Zorko, M. Gomilšek, M. Pregelj, K. W. Krämer, D. Biner, A. Biffin, C. Rüegg, and M. Klanjšek, Nat. Phys. 14, 786 (2018).

38 Y. Yu, Y. Xu, K. Ran, J. Ni, Y. Huang, J. Wang, J. Wen, and S. Li, Phys. Rev. Lett. 120, 067202 (2018).

39 R. Hentrich, A. U. B. Wolter, X. Zotos, W. Brenig, D. Nowak, A. Isaeva, T. Doert, A. Banerjee, P. Lampen- Kelley, D. G. Mandrus, S. E. Nagler, J. Sears, Y.-J. Kim, B. Büchner, and C. Hess, Phys. Rev. Lett. 120, 117204 (2018).

40 Y. Kasahara, T. Ohnishi, Y. Mizukami, O. Tanaka, S. Ma, K. Sugii, N. Kurita, H. Tanaka, J. Nasu, Y. Motome, T. Shibauchi and Y. Matsuda, Nature 559, 227 (2018).

41 J. S. Gordon, A. Catuneanu, E. S. Sørensen, and H.-Y. Kee, Nat. Commun. 10, 2470 (2019).

${ }^{42}$ Y. Motome, R. Sano, S. H. Jang, Y. Sugita, and Y. Kato, arXiv:2001.03731 (2020).

43 C. E. Agrapidis, J. van den Brink, and S. Nishimoto, Sci. Rep. 8, 1815 (2018).

44 W. Yang, A. Nocera, T. Tummuru, H.-Y. Kee, and I. Affleck, Phys. Rev. Lett. 124, 147205 (2020).

${ }^{45}$ W. Yang, A. Nocera, E. S. Sørensen, H.-Y. Kee, and I. Affleck, arXiv:2004.06074 (2020).

46 W. Yang, A. Nocera, and I. Affleck, arXiv:2004.12954 (2020).

47 C. E. Agrapidis, J. van den Brink, and S. Nishimoto, Phys. Rev. B 99, 224418 (2019).

48 A. Catuneanu, E. S. Sørensen, and H.-Y. Kee, Phys. Rev. B 99, 195112 (2019).

49 P. P. Stavropoulos, A. Catuneanu, and H.-Y. Kee, Phys. Rev. B 98, 104401 (2018).

50 S. R. White, Phys. Rev. Lett. 69, 2863 (1992).

51 S. R. White, Phys. Rev. B 48, 10345 (1993).

${ }^{52}$ R. J. de la Cruz and H. Faßbender, Linear Algebra and Its Applications 496, 288 (2016). 